\title{
A General Approach to Green Functors Using Bisets
}

\section{Laurence Barker}

To cite this article: Laurence Barker (2016) A General Approach to Green Functors Using Bisets, Communications in Algebra, 44:12, 5351-5375, DOI: 10.1080/00927872.2016.1172601

To link to this article: https://doi.org/10.1080/00927872.2016.1172601

\section{Published online: 06 Jul 2016.}

Submit your article to this journal ¿

Џlll Article views: 55

Q View related articles $\square$

View Crossmark data ¿ 


\section{A GENERAL APPROACH TO GREEN FUNCTORS USING BISETS}

Laurence Barker

Department of Mathematics, Bilkent University, Bilkent, Ankara, Turkey

We introduce a biset-theoretic notion of a Green functor which accommodates the functorial and ring-theoretic structural features of the modular character functor. For any Green functor $A$ in that sense, we introduce an algebra $\Lambda_{A}$. Any $\Lambda_{A}$-module has the same kind of functorial structure as $A$ and is also a module for the algebra $\bigoplus_{G} A(G)$, where $G$ runs over the underlying family of finite groups. In a globally defined scenario and also in a scenario localized to the subquotients of a fixed finite group, we take $A$ to be the modular character functor, and we classify the simple $\Lambda_{A}$-modules.

Key Words: Green biset functor; Green category; Modular character functor; Synthetic algebra.

2010 Mathematics Subject Classification: $\quad$ Primary: 20C20; Secondary: 19A22.

\section{INTRODUCTION}

Established notions in the theory of Mackey functors, biset functors and Green functors fail to capture all the fundamental structural features of the modular character ring. Let $\mathbb{O}$ be a complete discrete valuation ring such that the residue field $\mathbb{F}=\mathbb{O} / J(\mathbb{O})$ is of prime characteristic $p$ and the field of fractions $\mathbb{K}$ of $\mathbb{O}$ is algebraically closed and of characteristic zero. The Grothendieck ring $A_{\mathbb{F}}(G)$ of the category of $\mathbb{F} G$-modules coincides with the modular character ring of $\mathbb{F} G$ with character values in $\mathbb{K}$. We can form a functor $A_{\mathbb{F}}: G \mapsto A_{\mathbb{F}}(G)$ defined on a category whose objects are finite groups and whose morphisms are generated by inductions via injective group homomorphisms and restrictions via arbitrary group homomorphisms. In the terminology of the biset-theoretic approach to Mackey functors in Webb [6], $A_{\mathbb{F}}$ is a globally defined Mackey functor equipped with inflation maps but not with deflation maps. With that observation, however, we do not accommodate the fact that each $A_{\mathbb{F}}(G)$ is a ring.

In [1], Bouc introduced the notion of a Green biset functor and, for any Green biset functor $A$, the notion of an $A$-module. The $A$-modules can be identified with the modules of an algebra ${ }^{\oplus} A$. Those constructions depend on some strong hypotheses: The underlying family of finite groups must be closed under direct products; $A$ must be equipped with inductions via arbitrary group homomorphisms and restrictions via arbitrary group homomorphisms.

Received April 13, 2015; Revised December 11, 2015. Communicated by J. Zhang.

Address correspondence to Laurence Barker, Department of Mathematics, Bilkent University, 06800 Bilkent, Ankara, Turkey; E-mail: barker@fen.bilkent.edu.tr 
We shall introduce a general biset-theoretic notion of a Green functor and, in this general setting, we shall introduce a notion of a module associated with a Green functor. We shall realize $A_{\mathbb{F}}$ as a Green functor equipped with inflation maps. We shall do this in two different scenarios, one where the underlying family of finite groups includes representatives of all finite groups, the other where the underlying finite groups are the subquotients of a fixed finite group. In both scenarios, we shall classify the simple modules associated with the Green functor $\mathbb{K} A_{\mathbb{F}}$.

In Section 2, we shall define the notion of a Green category. Let $\mathfrak{K}$ be a set of finite groups such that, given $H \leq G \in \mathfrak{K}$, then $H \in \mathfrak{K}$. A Green category on $\mathfrak{K}$ is a certain kind of subcategory $\mathcal{G}$ of the biset category such that the set of objects is $\operatorname{obj}(\mathcal{G})=\mathfrak{K}$. Letting $R$ be a commutative unital ring, we can form the $R$-linear extension $R \mathcal{G}$ of $\mathcal{G}$. An $R \mathcal{G}$-functor is a functor $R \mathcal{G} \rightarrow R$-Mod. Equivalently, as we shall explain in Section 3, an $R \mathcal{G}$-functor is a module of an algebra ${ }^{\oplus} R \mathcal{G}$ called the quiver algebra of $R \mathcal{G}$. (The algebra ${ }^{\oplus} R \mathcal{G}$ is locally unital. All modules of locally unital rings are required to be locally unital. Section 3 for the terminology.) The motive for the definition of a Green category is that, in Section 3, we shall introduce the notion of a Green $R \mathcal{G}$-functor, which is an $R \mathcal{G}$-functor $A$ such that, for each element $G$ of the underlying family of finite groups, the $R$-module $A(G)$ is a ring satisfying two axiomatic conditions. One of the axioms says that restrictions act as ring homomorphisms. The other axiom is a variant of the familiar Frobenius relations for induction and restriction. The direct sum ${ }^{\Delta} A=\bigoplus_{G} A(G)$ is a module of two algebras, namely, ${ }^{\Delta} A$ itself and the quiver algebra ${ }^{\oplus} R \mathcal{G}$. As a synthesis of those two algebras, we shall form an algebra $\Lambda_{A}$, called the synthetic algebra of $A$, generated by an isomorphic copy of ${ }^{\Delta} A$ and a quotient of ${ }^{\oplus} R \mathcal{G}$.

The main purpose of Sections 4, 5, and 6 is to return to the scenario considered by Bouc and to compare the notion of a $\Lambda_{A}$-module with the notion of an $A$ module. Let $\mathfrak{L}$ be a set of finite groups such that, given $F, G \in \mathfrak{L}$, then $F \times G \in \mathfrak{L}$ and given $K \unlhd H \leq G$, then $H / K \in \mathfrak{L}$. Let $\mathcal{H}$ be the full subcategory of the biset category such that $\operatorname{obj}(\mathcal{H})=\mathfrak{L}$. In Section 4 , we shall review a theorem of Romero [4] asserting that the Green $R \mathcal{H}$-functors, as defined in this paper, coincide with the Green biset $R \mathcal{H}$-functors as defined by Bouc [1]. In Section 5, consolidating a result of Bouc [1] and Romero [4], we shall confirm that, for any $R \mathcal{H}$-functor $A$, the ${ }^{\oplus} A$ modules coincide with the $A$-modules. In Section 6 , we shall realize $\Lambda_{A}$ as a quotient algebra of ${ }^{\oplus} A$. Thus, every $\Lambda_{A}$-module becomes an $A$-module by inflation. Since the $A$-module ${ }^{\Delta} A$ is the inflation of a $\Lambda_{A}$-module, the problem of determining the $A$ module composition structure of ${ }^{\Delta} A$ is identical to the problem of determining the $\Lambda_{A}$-module structure of ${ }^{\Delta} A$.

Let $\mathfrak{K}_{\infty}$ be a set of finite groups such that $\mathfrak{K}_{\infty}$ is closed under taking subgroups, and every finite group is isomorphic to a group in $\mathfrak{K}_{\infty}$. For a fixed finite group $G$, let $\mathfrak{K}_{G}$ be the set of groups $H / K$ such that $K \unlhd H \leq G$. In Section 7, we shall consider two examples, one of them with $\mathfrak{K}=\mathfrak{K}_{\infty}$, the other with $\mathfrak{K}=\mathfrak{K}_{G}$. For both of the examples, we put $A=\mathbb{K} A_{\mathbb{F}}$. In those two cases, we shall classify the simple $\Lambda_{A}$-modules and we shall determine the composition structure of the $\Lambda_{A}$-module ${ }^{\Delta} A$.

One motive for this work is the speculation that, as a development of Brauer's induction theorem, a new decomposition of the modular character functor $A_{\mathbb{F}}$ may emerge from a study of the structure of ${ }^{\Delta} A_{\mathbb{F}}$ as a module of the integral synthetic algebra $\Lambda_{A_{\mathbb{F}}}$. We have examined the $\mathbb{K}$-linear extension as a step toward that goal. 


\section{GREEN CATEGORIES}

We shall introduce the notion of a Green category with set of objects $\mathfrak{K}$, where $\mathfrak{K}$ is as specified in Section 1. Our reason for working with a set of objects instead of a class of objects is that, in the next section, we shall be considering, for a given Green category, the quiver algebra of the Green category. The construction of the quiver algebra is valid only when the objects comprise a set. However, in applications, $\mathfrak{K}$ can play the role of a proper class by passage from a large category to an equivalent small category.

It is to be hoped that the reader is familiar with the theory of bisets, as described in Bouc [1, chapters 2, 3]. The biset category $\mathcal{C}$ is a linear category whose class of objects is the class of finite groups. Let $F, G, H$ be finite groups. The $\mathbb{Z}$ module of morphisms $F \leftarrow G$ in $\mathcal{C}$ is the Burnside ring $B(F, G)$ of $F \times G$. Given an $F$ - $G$-biset $X$, we write $[X]$ to denote the isomorphism class of $X$ as an element of $B(F, G)$. The composition in $\mathcal{C}$ is such that given an $F$ - $G$-biset $X$ and a $G-H$-biset $Y$, then the composite of $[X]$ and $[Y]$ is $[X][Y]=\left[X \times_{H} Y\right]$ where $X \times_{H} Y$ denotes the set of $H$-orbits in $X \times Y$. The identity morphism on $G$ is ${ }_{G}$ iso $_{G}=\left[{ }_{G}\right.$ Iso $\left._{G}\right]$ where ${ }_{G}$ Iso $_{G}={ }_{G} G_{G}=(G \times G) / \Delta(G)$ with ${ }_{G} G_{G}$, denoting $G$ as a $G$ - $G$-biset by left and right multiplication and $\Delta(G)=\{(g, g): g \in G\}$. (Note that we distinguish between the element ${ }_{G}$ iso $_{G} \in B(G, G)$ and the $G$ - $G$-biset ${ }_{G}$ Iso $_{G}$.)

The morphisms having the form $[(F \times G) / A]$, where $A \leq F \times G$, are called the transitive morphisms $F \leftarrow G$. When $F$ and $G$ are understood, we write the canonical projections as $p_{1}: F \leftarrow F \times G$ and $p_{2}: G \leftarrow F \times G$. For $A \leq F \times G$ and $B \leq G \times$ $H$, we define $A * B$ to be the subgroup of $F \times H$ consisting of those elements $(f, h)$ such that, for some $g \in G$, we have $(f, g) \in A$ and $(g, h) \in B$. The following characterization of the composition operation in $\mathcal{C}$ is $[1,2.3 .24]$.

Proposition 2.1 (Bouc.). Given finite groups $F, G, H$ and subgroups $A \leq F \times G$ and $B \leq G \times H$, then

$$
\left[\frac{F \times G}{A}\right]\left[\frac{G \times H}{B}\right]=\sum_{p_{2}(A) g p_{1}(B) \subseteq G}\left[\frac{F \times H}{A *{ }^{(g, 1)} B}\right]
$$

where, as the notation indicates, $g$ runs over $p_{1}(A)-p_{2}(B)$ double-coset representatives.

Given a group homomorphism $\alpha: F \leftarrow G$, we define transitive morphisms

$$
{ }_{F} \operatorname{ind}_{G}^{\alpha}=\left[\frac{F \times G}{\{(\alpha(g), g): g \in G\}}\right], \quad{ }_{G} \operatorname{res}_{F}^{\alpha}=\left[\frac{G \times F}{\{(g, \alpha(g)): g \in G\}}\right]
$$

called induction and restriction, respectively. Using the above proposition, it is easy to show that, given a group homomorphism $\beta: G \leftarrow H$, ind ${ }^{\alpha}$ ind $^{\beta}=$ ind $^{\alpha \beta}$ and $\operatorname{res}^{\beta} \operatorname{res}^{\alpha}=\operatorname{res}^{\alpha \beta}$.

Remark 2.2. Given a diagram $F \stackrel{\alpha}{\longleftarrow} I \stackrel{\theta}{\longrightarrow} G$ in the category of finite groups, then ${ }_{F} \operatorname{ind}_{I}^{\alpha} \operatorname{res}_{G}^{\theta}$ is a transitive morphism. Moreover, every transitive morphism in $\mathcal{C}$ has that form. 
Proof. By Proposition 2.1, ${ }_{F} \operatorname{ind}_{I}^{\alpha} \operatorname{res}_{G}^{\theta}=[(F \times G) /\{(\alpha(i), \theta(i)): i \in I\}]$. For $A \leq$ $F \times G$, we have $[(F \times G) / A]={ }_{F} \operatorname{ind}_{A}^{p_{1}} \operatorname{res}_{G}^{p_{2}}$.

The next result gives another formula for the product of two transitive morphisms.

Proposition 2.3. Given a diagram $F \stackrel{\alpha}{\longleftarrow} I \stackrel{\theta}{\longrightarrow} G \stackrel{\beta}{\longleftarrow} J \stackrel{\phi}{\longrightarrow} H$ in the category of finite groups, then

$$
{ }_{F} \operatorname{ind}_{I}^{\alpha} \operatorname{res}_{G}^{\theta} \operatorname{ind}_{J}^{\beta} \operatorname{res}_{H}^{\phi}=\sum_{\theta(I) g \beta(J) \subseteq G}{ }_{F} \operatorname{ind}_{L_{g}}^{\alpha \gamma_{g}} \operatorname{res}_{H}^{\phi \psi_{g}}
$$

where $I \stackrel{\gamma_{g}}{\longleftarrow} L_{g} \stackrel{\psi_{g}}{\longrightarrow} J$ is any pullback of $I \stackrel{\theta}{\longrightarrow} G \stackrel{g_{\beta}}{\longleftarrow} J$.

Proof. Putting $L_{g}=\{(i, j) \in I \times J: \theta(i)=\beta(j)\}$ and $\gamma_{g}(i, j)=i$ and $\psi_{g}(i, j)=j$, Proposition 2.1 and the proof of Remark 2.2 yield

$$
{ }_{I} \operatorname{res}_{G}^{\theta} \operatorname{ind}_{J}^{\beta}=\sum_{g}\left[\frac{I \times J}{L_{g}}\right]=\sum_{g}{ }_{I} \operatorname{ind}_{L_{g}}^{\gamma_{g}} \operatorname{res}_{J}^{\psi_{g}} .
$$

The group homomorphisms appearing in the latest proposition are illustrated in the next diagram. The square in the diagram is a pullback square.

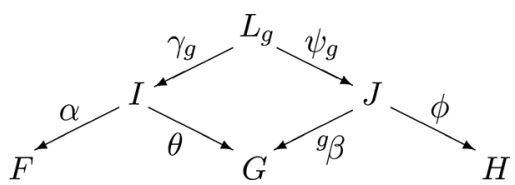

Recall, a category is said to be linear (or preadditive) provided the sets of morphisms are $\mathbb{Z}$-modules and composition is bilinear. Functors between linear categories are required to act on morphisms as linear maps.

We define a group category to be a linear subcategory $\mathcal{X}$ of $\mathcal{C}$ such that every morphism in $\mathcal{X}$ is a linear combination of transitive morphisms in $\mathcal{X}$. Given such $\mathcal{X}$ and $F, G \in \operatorname{obj}(\mathcal{X})$, we write $\mathcal{X}(F, G)$ to denote the $\mathbb{Z}$-module of morphisms $F \leftarrow G$ in $\mathcal{X}$.

We define a Mackey system on $\mathfrak{K}$ to be a subcategory $\mathcal{F}$ of the category of finite groups such that $\operatorname{obj}(\mathcal{F})=\mathfrak{K}$ and, writing $\mathcal{F}(F, G)$ for the set of morphisms to a group $F \in \mathfrak{K}$ from a group $G \in \mathfrak{K}$, the following four axioms hold:

MS1: For all $I \leq G \in \mathfrak{K}$, the inclusion $G \hookleftarrow I$ is in $\mathcal{F}(G, I)$.

MS2: For all $I \leq G \in \mathfrak{K}$ and $g \in G$, the conjugation map ${ }^{g} i \mapsto i$ is in $\mathcal{F}\left({ }^{g} I, I\right)$.

MS3: Given $\theta \in \mathcal{F}(F, G)$, then the restriction $\theta(G) \leftarrow G$ is in $\mathcal{F}(\theta(G), G)$.

MS4: Given $\theta \in \mathcal{F}(F, G)$ such that $\theta$ is a group isomorphism, then $\theta^{-1}$ is in $\mathcal{F}(G, F)$.

We mention that the notion of a Mackey system generalizes the notion of a fusion system. Indeed, given a $p$-group $P$, the Mackey systems on the set of subgroups of $P$ are precisely the fusion systems on $P$. 
Consider a pair of Mackey systems $(\mathcal{I}, \mathcal{R})$ such that the following three conditions hold:

GC1: The category $\mathcal{I}$ is a subcategory of $\mathcal{R}$.

GC2: Let $F \stackrel{\alpha}{\longleftarrow} I \stackrel{\theta}{\longrightarrow} G$ be a diagram with $\alpha \in \operatorname{mor}(\mathcal{I})$ and $\theta \in \operatorname{mor}(\mathcal{R})$. If $\theta$ is surjective and there exists a group homomorphism $\beta$ such that $\alpha=\beta \theta$, then $\beta \in \operatorname{mor}(\mathcal{I})$. If $\alpha$ is surjective and there exists a group homomorphism $\phi$ such that $\theta=\phi \alpha$, then $\phi \in \operatorname{mor}(\mathcal{R})$.

GC3: For every diagram $I \stackrel{\theta}{\longrightarrow} G \stackrel{\beta}{\longleftarrow} J$ with $\theta \in \operatorname{mor}(\mathcal{R})$ and $\beta \in \operatorname{mor}(\mathcal{I})$, there is a pullback $I \stackrel{\gamma}{\longleftarrow} L \stackrel{\psi}{\longrightarrow} J$ with $\gamma \in \operatorname{mor}(\mathcal{I})$ and $\psi \in \operatorname{mor}(\mathcal{R})$.

Let $\mathcal{G}$ be the linear subcategory of $\mathcal{C}$ such that $\operatorname{obj}(\mathcal{G})=\mathfrak{K}$ and the morphisms in $\mathcal{G}$ are generated by the morphisms ind $^{\alpha}$ and $\operatorname{res}^{\theta}$ where $\alpha \in \operatorname{mor}(\mathcal{I})$ and $\theta \in$ $\operatorname{mor}(\mathcal{R})$. We call $\mathcal{G}$ a Green category on $\mathfrak{K}$. We call the homomorphisms in $\mathcal{I}$ the induction homomorphisms for $\mathcal{G}$. We call the homomorphisms in $\mathcal{R}$ the restriction homomorphisms for $\mathcal{G}$. The motive for the somewhat technical axioms GC1, GC2, GC3 is that they will allow us to work productively with morphisms having the form $\operatorname{ind}^{\alpha} \operatorname{res}^{\theta}$ where $\alpha \in \operatorname{mor}(\mathcal{I})$ and $\theta \in \operatorname{mor}(\mathcal{R})$.

Proposition 2.4. Let $\mathcal{G}$ be a Green category on $\mathfrak{K}$. Then:

(1) $\mathcal{G}$ is a group category. The transitive morphisms in $\mathcal{G}$ are the morphisms ind ${ }^{\alpha} \mathrm{res}^{\theta}$ where $\alpha$ is an induction homomorphism for $\mathcal{G}$ and $\theta$ is a restriction homomorphism for $\mathcal{G}$.

(2) The induction homomorphisms for $\mathcal{G}$ are the homomorphisms $\beta$ such that $\mathrm{ind}^{\beta}$ is a morphism in $\mathcal{G}$. The restriction homomorphisms for $\mathcal{G}$ are the homomorphisms $\phi$ such that res $^{\phi}$ is a morphism in $\mathcal{G}$. In particular, $\mathcal{G}$ determines $\mathcal{I}$ and $\mathcal{R}$.

Proof. Plainly, the transitive morphisms of the specified form belong to $\mathcal{G}$. Via Proposition 2.3, condition GC3 ensures that every morphism in $\mathcal{G}$ is a linear combination of such transitive morphisms. We have established part (1). Trivially, if $\beta \in \operatorname{mor}(\mathcal{I})$, then $\operatorname{ind}^{\beta} \in \operatorname{mor}(\mathcal{G})$. Conversely, let $\beta: F \leftarrow G$ be a group homomorphism such that ${ }_{F} \operatorname{ind}_{G}^{\beta} \in \operatorname{mor}(\mathcal{G})$. By part $(1)$, there exist $I \in \mathfrak{K}$ and $\alpha \in$ $\mathcal{I}(F, I)$ and $\theta \in \mathcal{R}(G, I)$ such that ${ }_{F} \operatorname{ind}_{G}^{\beta}={ }_{F} \operatorname{ind}_{I}^{\alpha} \operatorname{res}_{G}^{\theta}$. We have

$$
[(F \times G) /\{(\beta(x), x): x \in G\}]=[(F \times G) /\{(\alpha(i), \theta(i)): i \in I\}] .
$$

Hence, $\theta$ is surjective and $\left\{\left({ }^{f} \beta(x),{ }^{g} x\right): x \in G\right\}=\{(\alpha(i), \theta(i)): i \in I\}$ for some $f \in F$ and $g \in G$. We have $\alpha=c(f) \beta c(g)^{-1} \theta$ where $c(f)$ and $c(g)$ denote the conjugation maps associated with $f$ and $g$. By condition GC2, $c(f) \beta c(g)^{-1} \in \operatorname{mor}(\mathcal{I})$. By the definition of a Mackey system, $c(f)^{-1}$ and $c(g)$ belong to $\operatorname{mor}(\mathcal{I})$. So the homomorphism $\beta=c(f)^{-1} c(f) \beta c(g)^{-1} c(g)$ belongs to $\operatorname{mor}(\mathcal{I})$. Half of part (2) is established. The other half of part (2) is similar.

In the latest proof, we did not use condition GC1. The motive for that condition comes from the Frobenius axiom in the definition of a Green functor in the next section. 
To present some examples of Green categories, some more notation will be convenient. Let $\alpha: F \leftarrow G$ be a group homomorphism. When $\alpha$ is surjective, we write $\operatorname{def}^{\alpha}=\operatorname{ind}^{\alpha}$ and inf ${ }^{\alpha}=\operatorname{res}^{\alpha}$, calling these morphisms deflation and inflation, respectively. When $\alpha$ is an isomorphism, we write iso ${ }^{\alpha}=$ ind $^{\alpha}=\operatorname{res}^{\alpha^{-1}}$, calling this morphism isogation. When $\alpha$ is an inclusion $F \hookleftarrow G$, we write ${ }_{F}$ ind $_{G}=$ ind $^{\alpha}$ and ${ }_{G} \operatorname{res}_{F}=\operatorname{res}^{\alpha}$. For arbitrary $\alpha$, we have

$$
{ }_{F} \operatorname{ind}_{G}^{\alpha}={ }_{F} \operatorname{ind}_{\alpha(G)} \text { iso }_{G / \operatorname{ker}(\alpha)}^{\alpha} \operatorname{def}_{G}, \quad{ }_{G} \operatorname{res}_{F}^{\alpha}={ }_{G} \underset{G / \operatorname{ker}(\alpha)}{\inf } \operatorname{iso}_{\alpha(G)}^{\alpha^{-1}} \operatorname{res}_{F} .
$$

For $A \leq F \times G$, we define $k_{2}(A)=\operatorname{ker}\left(p_{1}: F \leftarrow A\right)=\{g \in G:(1, g) \in A\}$. An $F-G$ biset $X$ is said to be right-free provided the action of $G$ is free. The next remark is easy to prove.

Remark 2.5. Given $A \leq F \times G$, then the following three conditions are equivalent: $k_{2}(A)=1$; the $F$ - $G$-biset $(F \times G) / A$ is right-free; there exist group homomorphisms $F \stackrel{\alpha}{\longleftarrow} I \stackrel{\theta}{\longrightarrow} G$, with $\alpha$ injective, such that $[(F \times G) / A]={ }_{F} \operatorname{ind}_{I}^{\alpha} \operatorname{res}_{G}^{\theta}$. In that case,

$$
[(F \times G) / A] \cong{ }_{F} \operatorname{ind}_{\alpha(I)} \operatorname{iso}_{I}^{\alpha} \inf _{\theta(I)}^{\theta} \operatorname{res}_{G} .
$$

For the next two examples, $\mathfrak{K}_{\infty}$ and $\mathfrak{K}_{G}$ are as specified in Section 1. The first one is suitable for dealing with functors on arbitrary finite groups in the presence of all inflations but no proper deflections.

Example 2.6. There is a Green category $\mathcal{G}_{\infty}$ on $\mathfrak{K}_{\infty}$ such that the restriction homomorphisms for $\mathcal{G}_{\infty}$ are the homomorphisms between groups in $\mathfrak{K}_{\infty}$ and the induction homomorphisms for $\mathcal{G}_{\infty}$ are the injective homomorphisms between groups in $\mathfrak{K}_{\infty}$. For $F, G \in \mathfrak{K}_{\infty}$, the $\mathbb{Z}$-module $\mathcal{G}_{\infty}(F, G)$ is spanned by the isomorphism of right-free $F$-G-bisets. The transitive morphisms in $\mathcal{G}_{\infty}$ have the form ${ }_{F} \operatorname{ind}_{I}^{\alpha} \operatorname{res}_{G}^{\theta}=$ ${ }_{F}$ ind $_{\alpha(I)}$ iso $_{I}^{\alpha} \inf _{\theta(I)}^{\theta}$ res $_{G}$ where $\alpha$ is injective.

Proof. Let $\mathcal{I}$ and $\mathcal{R}$ be the Mackey systems on $\mathfrak{K}$ such that $\operatorname{mor}(\mathcal{I})$ is the set of injective homomorphisms between groups in $\mathfrak{K}$ and $\operatorname{mor}(\mathcal{R})$ is the set of all homomorphisms between groups in $\mathfrak{K}$. Consider $\theta \in \operatorname{mor}(\mathcal{R})$ and $\beta \in \operatorname{mor}(\mathcal{I})$ such that $\theta$ and $\beta$ have the same codomain. Let $(\gamma, \psi)$ be a pullback of $(\theta, \beta)$ such that the domain of $\gamma$ and $\beta$ belongs to $\mathfrak{K}$. Since $\beta$ is injective, $\gamma$ is injective. So $(\mathcal{I}, \mathcal{R})$ satisfies condition GC3. Condition GC2 is easier to check and condition GC1 is trivial. The rest is clear using Proposition 2.4 and Remark 2.5.

The notion of a Green category is also applicable to scenarios associated with a fixed finite group. The next example is a finite analogue of the previous one and it is also an extension, with inflations, of the Green category implicit in Thévenaz [5]. Given finite groups $\underline{I} \unlhd I \leq G \geq K \unrhd \underline{K}$ and an element $u \in G$ such that ${ }^{u} I \leq K$ and ${ }^{u} \underline{I} \leq \underline{K}$, we define a homomorphism $c(u): I / \underline{I} \rightarrow K / \underline{K}$ such that $i \underline{I} \mapsto{ }^{u} i . \underline{K}$ for $i \in I$. We call $c(u)$ a conjugation map.

Example 2.7. Let $G$ be a finite group. Then there is a Green category $\mathcal{G}_{G}$ on $\mathfrak{K}_{G}$ such that the restriction homomorphisms for $\mathcal{G}_{G}$ are the conjugation maps and the 
induction homomorphisms for $\mathcal{G}_{G}$ are the injective conjugation maps. The transitive morphisms in $\mathcal{G}_{G}$ are the morphisms ${ }_{H / \underline{H}} \operatorname{ind}_{I / \underline{I}}^{c(a)} \operatorname{res}_{K / \underline{K}}^{c(u)}$ where $a, u \in G$ and $H \geq{ }^{a} I$, $\underline{H}={ }^{a} \underline{I},{ }^{u} I \leq K,{ }^{u} \underline{I} \leq \underline{K}$.

Proof. Let $\mathcal{I}$ and $\mathcal{R}$ be the Mackey systems on $\mathfrak{K}$ such that $\operatorname{mor}(\mathcal{I})$ is the set of injective conjugation maps and $\operatorname{mor}(\mathcal{R})$ is the set of all conjugation maps. Condition GC1 being trivial, we must check that $(\mathcal{I}, \mathcal{R})$ satisfies GC2 and GC3. Let $H / \underline{H} \stackrel{c(a)}{\longleftarrow} I / \underline{I} \stackrel{c(u)}{\longrightarrow} K / \underline{K}$ be a diagram with $c(a) \in \operatorname{mor}(\mathcal{I})$ and $c(u) \in \operatorname{mor}(\mathcal{R})$. If $c(u)$ is surjective and there exists a group homomorphism $\beta$ such that $c(a)=$ $\beta 。 c(u)$ then, bearing in mind that $c(a)$ is injective, $c(u)$ must be an isomorphism and $\beta=c\left(a u^{-1}\right)$. Half of condition GC2 has been confirmed. The other half of GC2 is similar.

Now let $J / \underline{J} \stackrel{c(u)}{\longrightarrow} H / \underline{H} \stackrel{c(b)}{\longleftarrow} K / \underline{K}$ be a diagram with $c(u) \in \operatorname{mor}(\mathcal{R})$ and $c(b) \in$ $\operatorname{mor}(\mathcal{I})$. Since $c(b)$ is injective, there is a pullback square as depicted, where $J^{\prime}=$ $J \cap u^{u^{-1}} K$. Condition GC3 has been confirmed. The last sentence of the assertion is clear.

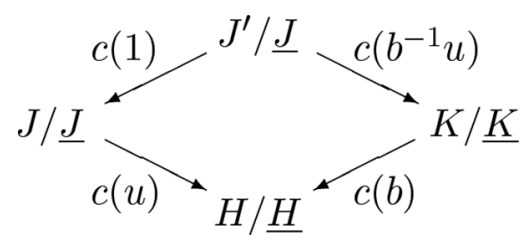

Note that, letting $\mathcal{I}=\mathcal{R}$ be the Mackey system on $\mathfrak{K}_{G}$ such that $\operatorname{mor}(\mathcal{I})=$ $\operatorname{mor}(\mathcal{R})$ is the set of all conjugation maps, then $(\mathcal{I}, \mathcal{R})$ fails condition GC3 except when $G$ is trivial. Indeed, the diagram $G \rightarrow 1 \leftarrow G$ has pullback group $G \times G$. Thus, it appears, Example 2.7 may indicate the closest one can get to formulating a good notion of a biset functor on a fixed finite group.

\section{GREEN FUNCTORS AND SYNTHETIC ALGEBRAS}

For any commutative unital ring $R$ and any Green category $\mathcal{G}$ on $\mathfrak{K}$, we can form the $R$-linear extension $R \mathcal{G}$, which we shall describe in a moment. In this section, we shall introduce the notion of a Green $R \mathcal{G}$-functor, which is a functor $A: R \mathcal{G} \rightarrow$ $R$-Mod such that, for each group $G$ in $\mathcal{G}$, the $R$-module $A(G)$ is equipped with a ring structure satisfying certain conditions. For any Green $R \mathcal{G}$-functor $A$, we shall construct an algebra $\Lambda_{A}$ called the synthetic algebra of $A$.

A category is said to be $R$-linear (or $R$-preadditive) provided the sets of morphisms are $R$-modules and the composition is $R$-bilinear. Functors between $R$ linear categories are required to act on morphisms as $R$-linear maps. We define $R \mathcal{G}$ to be the $R$-linear category such that $R \mathcal{G}(F, G)=R \otimes_{\mathbb{Z}} \mathcal{G}(F, G)$ for $F, G \in \mathfrak{K}$. Composition of morphisms in $R \mathcal{G}$ is by $R$-linear extension of the composition in $\mathcal{G}$.

Let us make some general comments about locally unital rings and $R$-linear functors. A ring $\Lambda$ is said to be locally unital provided, for all finite subsets $\Omega \subseteq \Lambda$, there exists an idempotent $i \in \Lambda$ such that $i \Omega i=\Omega$. A ring homomorphism between locally unital rings $\theta: \Lambda \rightarrow \Lambda^{\prime}$ is said to be locally unital provided, for all finite $\Omega \subseteq$ 
$\Lambda^{\prime}$, there exists an idempotent $i \in \Lambda$ such that $\theta(i) \Omega \theta(i)=\Omega$. For any ring $\Lambda$, a $\Lambda$ module $M$ is said to be locally unital provided, for all finite $\Omega \subseteq M$, there exists an idempotent $i \in \Lambda$ such that $i \Omega=\Omega$. To see a quick example, consider an infinite set $K$. For each $k \in K$, let $M_{k}$ be a nonzero unital module of a unital ring $\Lambda_{k}$. The direct sum $\Lambda=\bigoplus_{k} \Lambda_{k}$ is a locally unital ring, the direct sum $\bigoplus_{k} M_{k}$ is a locally unital $\Lambda$-module, but the direct product $\prod_{k} M_{k}$ is a $\Lambda$-module that is not locally unital. Henceforth, in this paper, all modules of locally unital rings are understood to be locally unital.

Consider a small $R$-linear category $\mathcal{L}$. We define an $\mathcal{L}$-functor to be a functor $\mathcal{L} \rightarrow R$-Mod. Let us explain how the $\mathcal{L}$-functors can be regarded as the modules. For $F, G \in \operatorname{obj}(\mathcal{L})$, we let $\mathcal{L}(F, G)$ denote the $R$-module of morphisms $F \leftarrow G$ in $\mathcal{L}$. We define the quiver algebra of $\mathcal{L}$ to be the locally unital algebra

$$
{ }^{\oplus} \mathcal{L}=\bigoplus_{F, G \in \operatorname{Obj}(\mathcal{L})} \mathcal{L}(F, G)
$$

whose multiplication is given by composition, products of incompatible morphisms being zero. The $\mathcal{L}$-functors can be identified with the ${ }^{\oplus} \mathcal{L}$-modules, each functor $G \mapsto$ $M(G)$ giving rise to a module $\bigoplus_{G} M(G)$, each module $M$ giving rise to functor $G \mapsto$ $\operatorname{id}_{G} M$.

Generalizing a definition implicit in Romero [4, Section 2], we define a Green $R \mathcal{G}$-functor to be an $R \mathcal{G}$-functor $A$ such that $A(G)$ is a unital $R$-algebra for each $G \in \mathfrak{K}$ and the following two axioms hold:

Restriction property: Given $I, G \in \mathfrak{K}$ and a restriction homomorphism $\theta: I \rightarrow G$ for $\mathcal{G}$, then ${ }_{I} \operatorname{res}_{G}^{\theta}: A(I) \leftarrow A(G)$ is a unital ring homomorphism.

Frobenius relations: Given $F, I \in \mathfrak{K}$, an induction homomorphism $\alpha: F \leftarrow I$ for $\mathcal{G}$ and elements $a \in A(F)$ and $b \in A(I)$, then

$$
{ }_{F} \operatorname{ind}_{I}^{\alpha}\left({ }_{I} \operatorname{res}_{F}^{\alpha}(a) \cdot b\right)=a \cdot{ }_{F} \operatorname{ind}_{I}^{\alpha}(b), \quad{ }_{F} \operatorname{ind}_{I}^{\alpha}\left(b \cdot{ }_{I} \operatorname{res}_{F}^{\alpha}(a)\right)={ }_{F} \operatorname{ind}_{I}^{\alpha}(b) \cdot a .
$$

Given Green $R \mathcal{G}$-functors $A$ and $A^{\prime}$, we define a morphism $f: A^{\prime} \leftarrow A$ to be a morphism of $R \mathcal{G}$-functors such that $f_{G}: A^{\prime}(G) \leftarrow A(G)$ is a ring homomorphism for each $G \in \mathfrak{K}$. Thus, we have specified the category of Green $R \mathcal{G}$-functors.

Let us note an example. Consider the Green functor $\mathcal{G}=\mathcal{G}_{\infty}$ as in Example 2.6. For each object $G \in \mathfrak{K}_{\infty}$, we can let $A(G)=R A_{\mathbb{F}}(G)$. For $F, G \in \mathfrak{K}_{\infty}$, and an $F$-G-biset $X$ such that $[X] \in \mathcal{G}(F, G)$, we can let $[X]$ act on $A(G)$ such that, writing $[[M]]$ to denote the Grothendieck equivalence class of an $\mathbb{F} G$-module $M$, we have $[X][[M]]=\left[\left[F X \otimes_{\mathbb{F} G} M\right]\right]$. To see that this action is well defined, note that, by Remark 2.5, $[X]$ is an $R$-linear combination of composites of inductions via inclusions, isogations, inflations, restrictions via inclusions. It is well known and easily checked that those four kinds of map are well defined on the modular character algebras $A(G)$, furthermore, the restriction property and the Frobenius relations hold. Thus, $A$ becomes a Green $R \mathcal{G}$-functor.

As another example, we can let $\mathcal{G}=\mathcal{G}_{G}$ as in Example 2.7, and we can again put $A=R A_{\mathbb{F}}(G)$. As two other examples, we can still let $\mathcal{G}$ be as in Example 2.6 or 2.6, and we can let $A$ be the cohomology ring $H^{*}(-, \mathbb{F})$ as an $\mathbb{F} \mathcal{G}$-functor. 
The next formula appears in Green [2, 1.84]. For arbitrary $\mathfrak{K}$ and $\mathcal{G}$, let $A$ be a Green $R \mathcal{G}$-functor. Let $G \in \mathfrak{K}$ and $U \leq G \geq V$. Let $u \in A(U)$ and $v \in A(V)$. Then

$$
{ }_{G} \operatorname{ind}_{U}(u) \cdot{ }_{G} \operatorname{ind}_{V}(v)=\sum_{U g V \subseteq G}{ }_{G} \operatorname{ind}_{U \cap^{g} V}\left({ }_{U \cap^{g} V} \operatorname{res}_{U}(u) \cdot{ }_{U \cap^{g} V} \operatorname{res}_{g_{V}} \operatorname{con}_{V}^{g}(v)\right)
$$

where ${ }_{{ }_{V}} \operatorname{con}_{V}^{g}={ }_{{ }_{V}}$ iso $_{V}^{c(g)}$. The context in [2] differs from ours, but the proof of the above version of the formula is still as in [2]; a straightforward application of the Mackey and Frobenius relations. Note that $U \hookleftarrow U \cap{ }^{g} V \stackrel{c\left(g^{-1}\right)}{\longrightarrow} V$ is a pullback of $U \hookrightarrow G \stackrel{c(g)}{\longleftarrow} V$. The following generalization of the formula will be used in the next section.

Proposition 3.1 (Mackey product formula). Let $A$ be a Green RG-functor. Let $\alpha$ : $G \leftarrow U$ and $\beta: G \leftarrow V$ be induction homomorphisms for $\mathcal{G}$. Let $u \in A(U)$ and $v \in$ $A(V)$. Then

$$
{ }_{G} \operatorname{ind}_{U}^{\alpha}(u) \cdot{ }_{G} \operatorname{ind}_{V}^{\beta}(v)=\sum_{\alpha(U) g \beta(V) \subseteq G}{ }_{G} \operatorname{ind}_{L_{g}}^{\alpha \gamma_{g}}\left(L_{g} \operatorname{res}_{U}^{\gamma_{g}}(u) \cdot{ }_{L_{g}} \operatorname{res}_{V}^{\eta_{g}}(v)\right)
$$

where $U \stackrel{\gamma_{g}}{\longleftarrow} L_{g} \stackrel{\eta_{g}}{\longrightarrow} V$ is any pullback of $U \stackrel{\alpha}{\longrightarrow} G \stackrel{\beta}{\longleftarrow} V$ such that $\gamma_{g}$ and $\eta_{g}$ are, respectively, an induction homomorphism and a restriction homomorphism for $\mathcal{G}$.

Proof. Using Proposition 2.3,

$$
{ }_{G} \operatorname{ind}_{U}^{\alpha}\left(u \cdot{ }_{U} \operatorname{res}_{G}^{\alpha} \operatorname{ind}_{V}^{\beta}(v)\right)=\sum_{\alpha(U) g \beta(V) \subseteq G}{ }_{G} \operatorname{ind}_{U}^{\alpha}\left(u \cdot{ }_{U} \operatorname{ind}_{L_{g}}^{\gamma_{g}} \operatorname{res}_{V}^{\eta_{g}}(v)\right)
$$

Two applications of the Frobenius relation complete the argument.

As algebras over $R$, we define

$$
{ }^{\Delta} A=\bigoplus_{G \in \mathfrak{K}} A(G), \quad E_{A}=\operatorname{End}_{R}\left({ }^{\Delta} A\right)=\bigoplus_{F, G \in \mathfrak{K}} E_{A}(F, G)
$$

where $E_{A}(F, G)$ is the $R$-module of $R$-maps $A(F) \leftarrow A(G)$. Let $\sigma$ and $v$ be the representations of ${ }^{\Delta} A$ as an $R \mathcal{G}$-module and as a ${ }^{\Delta} A$-module, respectively. Thus

$$
\sigma=\sigma_{A}:{ }^{\oplus} R \mathcal{G} \rightarrow E_{A}, \quad v=v_{A}:{ }^{\Delta} A \rightarrow E_{A}
$$

are the algebra maps such that $\sigma(x) b=x b$ and $v(b) b^{\prime}=b b^{\prime}$ for $x \in R \mathcal{G}(F, G)$ and $b, b^{\prime} \in A(G)$.

Lemma 3.2. Let $A$ be a Green $R \mathcal{G}$-functor, and let $F, G, I \in \mathfrak{K}$.

(1) Given $b \in A(G)$ and a restriction homomorphism $\theta: I \rightarrow G$ for $\mathcal{G}$, then

$$
\sigma\left({ }_{I} \operatorname{res}_{G}^{\theta}\right) v(b)=v\left({ }_{I} \operatorname{res}_{G}^{\theta}(b)\right) \sigma\left({ }_{I} \operatorname{res}_{G}^{\theta}\right) .
$$


(2) Given $a \in A(F), d \in A(I)$ and an induction homomorphism $\alpha: F \leftarrow I$ for $\mathcal{G}$, then

$$
v(a) \sigma\left({ }_{F} \operatorname{ind}_{I}^{\alpha}\right)=\sigma\left({ }_{F} \operatorname{ind}_{I}^{\alpha}\right) v\left({ }_{I} \operatorname{res}_{F}^{\alpha}(a)\right), \quad v\left({ }_{F} \operatorname{ind}_{I}^{\alpha}(d)\right)=\sigma\left({ }_{F} \operatorname{ind}_{I}^{\alpha}\right) v(d) \sigma\left({ }_{I} \operatorname{res}_{F}^{\alpha}\right) .
$$

Proof. For $b^{\prime} \in A(G)$, the restriction property yields

$$
\sigma\left({ }_{I} \operatorname{res}_{G}^{\theta}\right) v(b) b^{\prime}={ }_{I} \operatorname{res}_{G}^{\theta}\left(b b^{\prime}\right)={ }_{I} \operatorname{res}_{G}^{\theta}(b) \cdot{ }_{I} \operatorname{res}_{G}^{\theta}\left(b^{\prime}\right)=v\left({ }_{I} \operatorname{res}_{G}^{\theta}(b)\right) \sigma\left({ }_{I} \operatorname{res}_{G}^{\theta}\right) b^{\prime} .
$$

Part (2) can be demonstrated similarly using the Frobenius relations.

We define the synthetic algebra of $A$, denoted $\Lambda_{A}$, to be the subalgebra of $E_{A}$ generated by $\sigma\left({ }^{\oplus} R \mathcal{G}\right)$ and $v\left({ }^{\Delta} A\right)$. The next result gives a more explicit description of $\Lambda_{A}$.

Proposition 3.3. Let $A$ be a Green $R \mathcal{G}$-functor. Then

$$
\Lambda_{A}=\bigoplus_{F, G \in \mathfrak{K}} \Lambda_{A}(F, G)
$$

where $\Lambda_{A}(F, G)$ is the $R$-submodule of $E_{A}(F, G)$ spanned by the elements having the form $\sigma\left({ }_{F} \operatorname{ind}_{I}^{\alpha}\right) v(d) \sigma\left({ }_{I} \operatorname{res}_{G}^{\theta}\right)$ such that $\alpha: F \leftarrow I$ and $\theta: I \rightarrow G$ are, respectively, an induction homomorphism and a restriction homomorphism for $\mathcal{G}$ and $d \in A(I)$.

Proof. This follows from Lemma 3.2 and part (1) of Proposition 2.4.

\section{GREEN BISET FUNCTORS}

Let the set of finite groups $\mathfrak{L}$ and the Green category $\mathcal{H}$ be as specified in Section 1. Recall, $\mathfrak{L}$ is closed under direct products and subquotients, $\mathcal{H}$ is the full subcategory of the biset category such that the set of objects in $\mathcal{H}$ is $\mathcal{L}$. We shall show that the Green $R \mathcal{H}$-functors as defined in Section 2 coincide with the Green biset $R \mathcal{H}$-functors, we mean to say, the $R \mathcal{H}$-functors satisfying the conditions in Bouc [1, 8.5.1].

We begin by reviewing the conditions imposed by Bouc. After Bouc [1, 8.5.1], we define a Green biset $R \mathcal{H}$-functor to be an $R \mathcal{H}$-functor $\mathcal{A}$ equipped with an element $\epsilon^{\mathcal{A}} \in \mathcal{A}(1)$ and also equipped with, for each $F, G \in \mathfrak{L}$, an $R$-bilinear map $\mathcal{A}(F) \times$ $\mathcal{A}(G) \ni(a, b) \mapsto a \times b \in \mathcal{A}(F \times G)$, satisfying the following three conditions:

Associativity: Given $F, G, H \in \mathfrak{L}$ and $a \in \mathcal{A}(F), b \in \mathcal{A}(G), c \in \mathcal{A}(H)$ then, via the canonical isomorphism $(F \times G) \times H \leftarrow F \times(G \times H)$, we have $(a \times b) \times c=$ $(F \times G) \times H$ iso $_{F \times(G \times H)}(a \times(b \times c))$.

Identity: Given $G \in \mathfrak{L}$ and $b \in \mathcal{A}(G)$ then, via the canonical isomorphisms $G \leftarrow 1 \times$ $G$ and $G \leftarrow G \times 1$, we have ${ }_{G}$ iso $_{1 \times G}\left(\epsilon^{\mathcal{A}} \times b\right)=b={ }_{G}$ iso $_{G \times 1}\left(b \times \epsilon^{\mathcal{A}}\right)$.

Functorality: Given morphisms $x: F^{\prime} \leftarrow F$ and $y: G^{\prime} \leftarrow G$ in $R \mathcal{H}$ and $a \in \mathcal{A}(F), b \in$ $\mathcal{A}(G)$, then $(x \times y)(a \times b)=x a \times y b$. 
Given Green biset $R \mathcal{H}$-functors $\mathcal{A}$ and $\mathcal{A}^{\prime}$, we define a morphism $f: \mathcal{A}^{\prime} \leftarrow \mathcal{A}$ to be a morphism of $R \mathcal{H}$-functors such that $f_{F \times G}(a \times b)=f_{F}(a) \times f_{G}(b)$ for $F, G \in \mathfrak{L}$ and $a \in \mathcal{A}(F), b \in \mathcal{A}(G)$, Thus, we obtain a category of Green biset $R \mathcal{H}$-functors.

We define an $R$-bilinear map $\mathcal{A}(G) \times \mathcal{A}(G) \ni\left(b, b^{\prime}\right) \mapsto b b^{\prime} \in \mathcal{A}(G)$ such that

$$
b b^{\prime}={ }_{G} \operatorname{res}_{G \times G}^{\delta_{G}}\left(b \times b^{\prime}\right)
$$

where $\delta_{G}: G \ni g \mapsto(g, g) \in G \times G$. We define $\epsilon_{G}^{\mathcal{A}}={ }_{G} \inf _{1}\left(\epsilon^{\mathcal{A}}\right)$.

Theorem 4.1. Let $\mathcal{A}$ be a Green biset RH-functor. Equipped with the above multiplication operation, $\mathcal{A}(G)$ becomes an algebra over $R$ with unity element $\epsilon_{G}^{\mathcal{A}}$ for each $G \in \mathfrak{L}$. Equipped with those algebra structures, $\mathcal{A}$ becomes a Green $R \mathcal{H}$-functor.

Proof. We have associativity $b\left(b^{\prime} b^{\prime \prime}\right)=\left(b b^{\prime}\right) b^{\prime \prime}$ because, by straightforward calculations,

$$
\left[\frac{G \times(G \times(G \times G))}{\{(g,(g,(g, g)))\}}\right]\left(b \times\left(b^{\prime} \times b^{\prime \prime}\right)\right)=\left[\frac{G \times((G \times G) \times G)}{\{(g,((g, g), g))\}}\right]\left(\left(b \times b^{\prime}\right) \times b^{\prime \prime}\right)
$$

where $b, b^{\prime}, b^{\prime \prime} \in \mathcal{A}(G)$ and $g$ runs over the elements of $G$. We have

$$
b \times \epsilon_{G}^{\mathcal{A}}=\left({ }_{G} \text { iso }_{G} \times{ }_{G} \inf _{1}\right)\left(b \times \epsilon^{\mathcal{A}}\right)={ }_{G \times G} \inf _{G \times 1}\left(b \times \epsilon^{\mathcal{A}}\right)={ }_{G \times G} \inf _{G \times 1} \operatorname{iso}_{G}(b) .
$$

Hence $b \epsilon_{G}^{\mathcal{A}}={ }_{G} \operatorname{res}_{G \times G}^{\delta_{G}}\left(b \times \epsilon_{G}^{\mathcal{A}}\right)={ }_{G} \operatorname{res}_{G \times G}^{\delta_{G}} \inf { }_{G \times 1} \operatorname{iso}_{G}(b)={ }_{G}$ iso $_{G}(b)=b$. Similarly, $\epsilon_{G}^{\mathcal{A}} b=b$. All the other axioms of a unital algebra are easy to check.

Let $I, G, \theta$ be as in the hypothesis of the restriction axiom. Given $b, b^{\prime} \in \mathcal{A}(G)$, then

${ }_{I} \operatorname{res}_{G}^{\theta}(b) \cdot{ }_{I} \operatorname{res}_{G}^{\theta}\left(b^{\prime}\right)={ }_{I} \operatorname{res}_{I \times I}^{\delta_{I}}\left({ }_{I} \operatorname{res}_{G}^{\theta} \times{ }_{I} \operatorname{res}_{G}^{\theta}\right)\left(b \times b^{\prime}\right)={ }_{I} \operatorname{res}_{G}^{\theta} \operatorname{res}_{G \times G}^{\delta_{G}}\left(b \times b^{\prime}\right)={ }_{I} \operatorname{res}_{G}^{\theta}\left(b b^{\prime}\right)$.

Since ${ }_{I} \operatorname{res}_{G}^{\theta} \inf _{1}={ }_{I} \inf _{1}$, we have ${ }_{I} \operatorname{res}_{G}^{\theta}\left(\epsilon_{G}^{\mathcal{A}}\right)=\epsilon_{I}^{G}$. We have confirmed the restriction axiom. Let $F, I, \alpha, a, b$ be as in the hypothesis of the Frobenius axiom. Then

$$
{ }_{F} \operatorname{ind}_{I}^{\alpha} \operatorname{res}_{I \times I}^{\delta_{I}}\left({ }_{I} \operatorname{res}_{F}^{\theta} \times{ }_{I} \text { iso }_{I}\right)=\left[\frac{F \times(F \times I)}{\{(\alpha(i),(\alpha(i), i))\}}\right]={ }_{F} \operatorname{res}_{F \times F}^{\delta_{F}}\left({ }_{F} \operatorname{iso}_{F} \times{ }_{F} \operatorname{ind}_{I}^{\alpha}\right)
$$

where $i$ runs over the elements of $I$. Applying this morphism to $a \times b$, we obtain one of the two Frobenius relations. The other is obtained similarly.

Romero [4, Section 2] has already noted how any Green $R \mathcal{H}$-functor $A$ can be regarded as a Green biset $R \mathcal{H}$-functor. The result was tangential to her concerns but it is crucial to ours. We shall give a detailed proof that the constructed functor satisfies the functorality axiom. After Romero, given $F, G \in \mathfrak{L}$, we define an $R$ bilinear map $A(F) \times A(G) \ni(a, b) \mapsto a \times b \in A(F \times G)$ such that

$$
a \times b={ }_{F \times G} \inf _{F}^{p_{1}}(a) \cdot_{F \times G} \inf _{G}^{p_{2}}(b) .
$$


Lemma 4.2. With the notation above, letting $F^{\prime}, G^{\prime} \in \mathfrak{L}$ and letting $\theta: F^{\prime} \rightarrow F$ and $\phi: G^{\prime} \rightarrow G$ be homomorphisms, then

$$
{ }_{F^{\prime} \times G^{\prime}} \operatorname{res}_{F \times G}^{\theta \times \phi}(a \times b)={ }_{F^{\prime}} \operatorname{res}_{F}^{\theta}(a) \times{ }_{G^{\prime}} \operatorname{res}_{G}^{\phi}(b) .
$$

Proof. Using the restriction axiom, the required equality reduces to

$$
F_{F^{\prime} \times G^{\prime}} \operatorname{res}_{F \times G}^{\theta \times \phi} \inf _{G}^{p_{1}}(a) \cdot_{F^{\prime} \times G^{\prime}} \operatorname{res}_{F \times G}^{\theta \times \phi} \inf _{G}^{p_{2}}(b)={ }_{F^{\prime} \times G^{\prime}} \inf _{F^{\prime}}^{p_{1}} \operatorname{res}_{F}^{\theta}(a) \cdot_{F^{\prime} \times G^{\prime}} \inf _{G^{\prime}}^{p_{2}} \operatorname{res}_{G}^{\phi}(b) .
$$

Lemma 4.3. With the notation above, letting $\alpha: F^{\prime} \leftarrow F$ and $\beta: G^{\prime} \leftarrow G$ be homomorphisms, then

$$
{ }_{F^{\prime} \times G^{\prime}} \operatorname{ind}_{F \times G}^{\alpha \times \beta}(a \times b)={ }_{F^{\prime}} \operatorname{ind}_{F}^{\alpha}(a) \times{ }_{G^{\prime}} \operatorname{ind}_{G}^{\beta}(b) .
$$

Proof. Using Proposition 2.1, ${ }_{F^{\prime} \times G^{\prime}} \inf _{F^{\prime}}^{p_{1}} \operatorname{ind}_{F}^{\alpha}={ }_{F^{\prime} \times G^{\prime}} \operatorname{ind}_{F \times G^{\prime}}^{\alpha \times 1} \inf _{F}^{p_{1}}$ and similarly for $\beta$. So, letting $x={ }_{F^{\prime}}$ ind $\alpha$ and $y={ }_{G^{\prime}}$ ind $_{G}^{\beta}$, we have

$$
x a \times y b={ }_{F^{\prime} \times G^{\prime}} \operatorname{ind}_{F \times G^{\prime}}^{\alpha \times 1}(u) \cdot_{F^{\prime} \times G^{\prime}} \operatorname{ind}_{F^{\prime} \times G}^{1 \times \beta}(v)
$$

where $u={ }_{F \times G^{\prime}} \inf _{F}^{p_{1}}(a)$ and $v={ }_{F^{\prime} \times G} \inf _{G}^{p_{2}}(b)$. Noting that $F \times G^{\prime} \stackrel{1 \times \beta}{\longleftarrow} F \times G \stackrel{\alpha \times 1}{\longrightarrow}$ $F^{\prime} \times G$ is a pullback of $F \times G^{\prime} \stackrel{\alpha \times 1}{\longrightarrow} F^{\prime} \times G^{\prime} \stackrel{1 \times \beta}{\longleftarrow} F^{\prime} \times G$, Proposition 3.1 yields

$$
x a \times y b={ }_{F^{\prime} \times G^{\prime}} \operatorname{ind}_{F \times G}^{\alpha \times \beta}\left({ }_{F \times G} \operatorname{res}_{F \times G^{\prime}}^{1 \times \beta}(u) \cdot_{F \times G} \operatorname{res}_{F^{\prime} \times G}^{\alpha \times 1}(v)\right) .
$$

By Proposition 2.1 again, ${ }_{F \times G} \operatorname{res}_{F \times G^{\prime}}^{1 \times \beta}(u)={ }_{F \times G} \inf _{F}^{p_{1}}(a)$ and similarly for $\alpha$ and $v$. Therefore, $x a \times y b=(x \times y)(a \times b)$.

Theorem 4.4 (Romero.). Let $A$ be a Green RH-functor. Equipping A with the above operation $\times$, letting $\epsilon^{A}$ be the unity element of $A(1)$, then $A$ becomes a multiplicative $R \mathcal{H}$-functor.

Proof. Using the restriction axiom, straightforward manipulations show that $A$ satisfies the associativity and identity axioms. Let $x, y, a, b$ be as in the hypothesis of the functorality axiom. To deduce the relation $(x \times y)(a \times b)=x a \times y b$, we many assume that $x$ and $y$ are transitive. Then $x$ and $y$ have the form specified in part (1) of Proposition 2.4. The required relation now follows from the latest two lemmas.

The constructions in Theorems 4.1 and 4.4 are mutually inverse. On a Green biset $R \mathcal{H}$-functor $\mathcal{A}$, we can impose the structure of a Green $R \mathcal{H}$-functor as in Theorem 4.1, then we can impose the structure of a Green biset $R \mathcal{H}$-functor as in Theorem 4.4. It is easy to check that the Green biset $R \mathcal{H}$-functor thus constructed coincides with $\mathcal{A}$. Likewise, given a Green $R \mathcal{H}$-functor $A$, we can construct a Green biset $R \mathcal{H}$-functor and then construct a Green $R \mathcal{H}$-functor. Again, it is easy to check that the two Green $R \mathcal{H}$-functors coincide. 
Given a morphism $f: \mathcal{A}^{\prime} \leftarrow \mathcal{A}$ of Green biset $R \mathcal{H}$-functors, then

$$
f_{G}\left({ }_{G} \operatorname{res}_{G \times G}^{\delta_{G}}\left(b \times b^{\prime}\right)\right)={ }_{G} \operatorname{res}_{G \times G}^{\delta_{G}}\left(f_{G \times G}\left(b \times b^{\prime}\right)\right)={ }_{G} \operatorname{res}_{G \times G}^{\delta_{G}}\left(f_{G}(b) \times f_{G}\left(b^{\prime}\right)\right)
$$

for all $G \in \mathfrak{L}$ and $b, b^{\prime} \in \mathcal{A}(G)$. Hence, regarding $\mathcal{A}$ and $\mathcal{A}^{\prime}$ as Green $R \mathcal{H}$-functors, $f_{G}\left(b b^{\prime}\right)=f_{G}(b) f_{G}\left(b^{\prime}\right)$, in other words, $f$ is a morphism of Green $R \mathcal{H}$-functors. It is easy to check that, conversely, any morphism of Green $R \mathcal{H}$-functors is a morphism of Green biset $R \mathcal{H}$-functors. Therefore, the category of Green biset $R \mathcal{H}$-functors is equivalent to the category of Green $R \mathcal{H}$-functors. In fact, we have explained how the Green biset $R \mathcal{H}$-functors can be identified with the Green $R \mathcal{H}$-functors.

\section{MODULES OF GREEN FUNCTORS}

We still let $\mathfrak{L}$ and $\mathcal{H}$ be as in Section 1 . For a Green $R \mathcal{H}$-functor $A$, we shall introduce an algebra ${ }^{\oplus} A$. After showing that ${ }^{\oplus} A$-modules satisfy versions of the restriction property, the Frobenius relations and the Mackey product formula, we shall recover a result of Bouc $[1,8.6 .1]$ and Romero $[4,2.11]$ which, reinterpreted, says that the ${ }^{\oplus} A$-modules coincide with the $A$-modules in the sense of $[1,8.5 .5]$.

Thanks to Theorem 4.4, we can understand $A$ to be equipped with the operation $\times$ as defined in the previous section. The operations $\left(b, b^{\prime}\right) \mapsto b b^{\prime}$ and $(a, b) \mapsto a \times b$ determine each other via the equalities

$$
b b^{\prime}={ }_{G} \operatorname{res}_{G \times G}^{\delta_{G}}\left(b \times b^{\prime}\right), \quad a \times b={ }_{F \times G} \inf _{F}^{p_{1}(a)} \cdot{ }_{F \times G} \inf _{G}^{p_{2}}(b) .
$$

where $b, b^{\prime} \in A(G)$ and $a \in A(F)$. As noted in Theorem 4.1, the unity element of $A(G)$ is $\epsilon_{G}^{A}={ }_{G} \inf _{1}\left(\epsilon^{A}\right)$. Given $F, G, H \in \mathfrak{L}$ then, as an element of $B(F, H)$, we define

${ }_{F \times H} \operatorname{des}_{(F \times G) \times(G \times H)}={ }_{F \times H} \operatorname{def}_{F \times \Delta(G) \times H} \operatorname{res}_{(F \times G) \times(G \times H)}=\left[\frac{(F \times H) \times((F \times G) \times(G \times H))}{\{((f, h),((f, g),(g, h)))\}}\right]$

running over $f \in F, g \in G, h \in H$. In the notation of Bouc [1, 2.3.9, 2.3.13],

$$
\left.{ }_{F \times H} \operatorname{des}_{(F \times G) \times(G \times H)}=\left[\operatorname{Iso}_{(F \times \Delta(G) \times H) / \Delta(G) \circ}^{F \times H} \circ \operatorname{Defres}_{(F \times \Delta(G) \times G) / \Delta(G)}^{(F \times G) \times(G \times H)}\right)\right] .
$$

Let $A(F, G)=A(F \times G)$. Following Bouc [1, 8.6.1], we define an $R$-bilinear map $A(F, G) \times A(G, H) \ni(u, v) \mapsto u_{\circ} v \in A(F, H)$ given by

$$
u_{\circ} v={ }_{F \times H} \operatorname{des}_{(F \times G) \times(G \times H)}(u \times v) .
$$

Let $K \in \mathfrak{L}$ and $w \in A(H, K)$. Omitting easy details,

$$
\begin{aligned}
u_{\circ}(v \circ w) & ={ }_{F \times K} \operatorname{des}_{(F \times G) \times(G \times H)}(\text { iso } \times \operatorname{des})_{(F \times G) \times((G \times H) \times(H \times K))}(u \times(v \times w)) \\
& ={ }_{F \times K} \operatorname{des}_{(F \times H) \times(H \times K)}(\operatorname{des} \times \text { iso })_{((F \times G) \times(G \times H)) \times(H \times K)}((u \times v) \times w)=(u \circ v)_{\circ} w .
\end{aligned}
$$

It is also easy to show that $u_{\circ} \epsilon_{G}^{A}=u$ and $\epsilon_{G \circ}^{A} 。=v$. So there is an $R$-linear category $\mathcal{P}_{A}$ with set of objects $\mathfrak{L}$, the $R$-module of morphisms $F \leftarrow G$ being $A(F, G)$, the 
composition operation being the above map $(u, v) \mapsto u_{\circ} v$. The quiver algebra of $\mathcal{P}_{A}$ is the $R$-module

$$
{ }^{\oplus} A=\bigoplus_{F, G \in \mathfrak{L}} A(F, G)
$$

equipped with the multiplication operation coming from composition. To avoid confusion with the multiplication operation ${ }^{\Delta} A \times{ }^{\Delta} A \ni(s, t) \mapsto s t \in{ }^{\Delta} A$, we shall always write the multiplication operation on ${ }^{\oplus} A$ as ${ }^{\oplus} A \times{ }^{\oplus} A \ni(s, t) \mapsto s_{\circ} t \in{ }^{\oplus} A$.

As explained in Section 2 for $R$-linear categories in general, the functors $\mathcal{P}_{A} \rightarrow$ $R$-Mod can be identified with the ${ }^{\oplus} A$-modules. The identification is such that, for an ${ }^{\oplus} A$-module $M$, we can write $M=\bigoplus_{G} M(G)$ and $M(G)=\mathrm{id}_{G}^{A} \cdot M$, where $\mathrm{id}_{G}^{A}$ is the identity morphism on $G$ in $\mathcal{P}_{A}$, we mean, $\mathrm{id}_{G}^{A}$ is the unity element of the algebra $A(G, G)=\operatorname{End}_{\mathcal{P}_{A}}(G)$.

As an element of $B(G \times G, 1)$, we define

$$
{ }_{G \times G} \operatorname{tin}_{1}={ }_{G \times G} \operatorname{ind}_{G}^{\delta_{G}} \inf _{1}=\left[\frac{(G \times G) \times 1}{\{((g, g), 1)\}}\right]
$$

where $g$ runs over the elements of $G$. In the notation of Bouc $[1,2.3 .9,2.3 .13,8.2 .6]$,

$$
{ }_{G \times G} \operatorname{tin}_{1}=\left[\operatorname{Indinf}_{\Delta(G) / \Delta(G) \circ}^{G \times G} \operatorname{Iso}_{1}^{\Delta(G) / \Delta(G)}\right]=[\vec{G}] .
$$

Lemma 5.1. With the notation above, ${ }_{G \times G} \operatorname{tin}_{1}\left(\epsilon^{A}\right)=\mathrm{id}_{G}^{A}$.

Proof. Let $t={ }_{G \times G} \operatorname{tin}_{1}\left(\epsilon^{A}\right)$. The identity axiom yields

$$
t_{\circ} b={ }_{G \times G} \operatorname{des}_{(G \times G) \times(G \times G)}(\operatorname{tin} \times \text { iso })_{1 \times(G \times G)}\left(\epsilon^{A} \times b\right)
$$

for all $b \in A(G)$. By direct calculation, $t_{\circ} b=b$. Similarly, $b_{\circ} t=b$. map

The quiver algebra of $R \mathcal{H}$ is ${ }^{\oplus} R \mathcal{H}=\bigoplus_{F, G \in \mathfrak{L}} R B(F, G)$. We define an $R$-linear

$$
\hat{\sigma}=\hat{\sigma}_{A}:{ }^{\oplus} R \mathcal{H} \rightarrow{ }^{\oplus} A
$$

which restricts to maps $R B(F, G) \rightarrow A(F, G)$ such that, given $x \in R B(F, G)$, then

$$
\hat{\sigma}(x)=\left({ }_{(F \times G) \times 1} \operatorname{iso}_{F \times G}(x)\right) \epsilon^{A} .
$$

The formula makes sense because the element ${ }_{(F \times G) \times 1}$ iso $_{F \times G}(x)$ of $R B(F \times G, 1)$ sends the element $\epsilon^{A}$ of $A(1)$ to an element of $A(F, G)$. We mention that the above maps $R B(F, G) \rightarrow A(F, G)$ coincide with the evaluations $R B(F \times G) \rightarrow A(F \times G)$ of the morphism denoted $e: R B \rightarrow A$ in Bouc [1, 8.5.1].

Lemma 5.2. With the notation above, $\hat{\sigma}$ is a locally unital algebra map and $\hat{\sigma}\left({ }_{G} \mathrm{iso}_{G}\right)=\mathrm{id}_{G}^{A}$. 
Proof. Let $X={ }_{F} X_{G}$ be an $F$-G-biset. Writing ${ }_{F \times G} X_{1}$ to denote $X$ as an $F \times G-1$ biset, then $\left[{ }_{F \times G} X_{1}\right]={ }_{(F \times G) \times 1}$ iso $_{F \times G}\left[{ }_{F} X_{G}\right]$. Let $Y={ }_{G} Y_{H}$ be a $G-H$-biset. We have

$$
\hat{\sigma}\left[{ }_{F} X_{G}\right]_{\circ} \hat{\sigma}\left[{ }_{G} Y_{H}\right]={ }_{F \times H} \operatorname{des}_{(F \times G) \times(G \times H)}\left(\left[{ }_{F \times G} X_{1}\right] \times\left[{ }_{G \times H} Y_{1}\right]\right)_{1 \times 1} \text { iso }_{1}\left(\epsilon^{A}\right)
$$

by the identity and functorality axioms for $A$. Restricting $X \times Y$ to $F \times \Delta(G) \times H$ and then deflating to $F \times H$, we obtain the $F \times H$-set of $G$-orbits $X \times{ }_{G} Y$. So

$$
\hat{\sigma}\left[{ }_{F} X_{G}\right]_{\circ} \hat{\sigma}\left[{ }_{G} Y_{H}\right]=\left[{ }_{F \times H}\left(X \times{ }_{G} Y\right)_{1}\right] \epsilon^{A}=\hat{\sigma}\left(\left[{ }_{F} X_{G}\right]\left[{ }_{G} Y_{H}\right]\right) .
$$

Therefore $\hat{\sigma}$ is an algebra map. We have ${ }_{(G \times G) \times 1}$ iso $_{G \times G}\left({ }_{G}\right.$ iso $\left._{G}\right)={ }_{G \times G} \operatorname{tin}_{1}$. Applying Lemma 5.1, $\hat{\sigma}\left({ }_{G}\right.$ iso $\left._{G}\right)={ }_{G \times G} \operatorname{tin}_{1}\left(\epsilon^{A}\right)=\mathrm{id}_{G}^{A}$. In particular, the algebra map $\hat{\sigma}$ is locally unital.

We define an $R$-linear map

$$
\hat{v}=\hat{v}_{A}:{ }^{\Delta} A \rightarrow{ }^{\oplus} A
$$

which restricts to maps $A(G) \rightarrow A(G, G)$ such that, for $b \in A(G)$, we have

$$
\hat{v}(b)={ }_{G \times G} \operatorname{ind}_{G}^{\delta_{G}}(b) .
$$

Lemma 5.3. With the notation above, $\hat{v}$ is a locally unital algebra map and $\hat{v}\left(\epsilon_{G}^{A}\right)=\mathrm{id}_{G}^{A}$.

Proof. A routine calculation, details omitted, shows that

$$
\hat{v}(b) \circ \hat{v}\left(b^{\prime}\right)=[((G \times G) \times(G \times G)) / \Delta(\Delta(G))]=\hat{v}\left(b b^{\prime}\right)
$$

for $b, b^{\prime} \in A(G)$. Lemma 5.1 and the definition of $\epsilon_{G}^{A}$ yield $\hat{v}\left(\epsilon_{G}^{A}\right)=\mathrm{id}_{G}^{A}$.

Lemma 5.4. With the notation above, given $u \in A(F, G)$, then

$$
u=\hat{\sigma}\left({ }_{F} \operatorname{def}_{F \times G}^{p_{1}}\right)_{\circ} \hat{v}(u)_{\circ} \hat{\sigma}\left({ }_{F \times G} \inf _{G}^{p_{2}}\right) .
$$

In particular, ${ }^{\oplus} A$ is generated by the subalgebras $\hat{\sigma}\left({ }^{\oplus} R \mathcal{H}\right)$ and $\hat{v}\left({ }^{\Delta} A\right)$.

Proof. Using the identity and functorality properties of $A$, together with Proposition 2.1, a long but straightforward calculation yields

$$
\left.\hat{v}(u) 。 \hat{\sigma}(F \times G) \inf _{G}^{p_{2}}\right)=\left[\frac{((F \times G) \times G) \times(F \times G)}{\{(((f, g), g)(f, g))\}}\right] u
$$

running over $f \in F$ and $g \in G$. A similar calculation then yields the required equality.

We have the following analogue of Lemma 3.2. 
Lemma 5.5. Let $F, G, I \in \mathfrak{L}$.

(1) Given $b \in A(G)$ and a homomorphism $\theta: I \rightarrow G$, then

$$
\hat{\sigma}\left({ }_{I} \operatorname{res}_{G}^{\theta}\right)_{\circ} \hat{v}(b)=\hat{v}\left({ }_{I} \operatorname{res}_{G}^{\theta}(b)\right)_{\circ} \hat{\sigma}\left({ }_{I} \operatorname{res}_{G}^{\theta}\right) .
$$

(2) Given $a \in A(F), d \in A(I)$ and a homomorphism $\alpha: F \leftarrow I$, then

$$
\hat{v}(a)_{\circ} \hat{\sigma}\left({ }_{F} \operatorname{ind}_{I}^{\alpha}\right)=\hat{\sigma}\left({ }_{F} \operatorname{ind}_{I}^{\alpha}\right)_{\circ} \hat{v}\left({ }_{I} \operatorname{res}_{F}^{\alpha}(a)\right), \quad \hat{v}\left({ }_{F} \operatorname{ind}_{I}^{\alpha}(d)\right)=\hat{\sigma}\left({ }_{F} \operatorname{ind}_{I}^{\alpha}\right)_{\circ} \hat{v}(d) \circ \hat{\sigma}\left({ }_{I} \operatorname{res}_{F}^{\alpha}\right) .
$$

Proof. The left-hand side of the equality asserted in part (1) is

$$
\begin{aligned}
I_{I \times G} \operatorname{des}_{(I \times G) \times(G \times G)}\left(\left[\frac{(I \times G) \times 1}{\{((i, \theta(i)), 1)\}}\right] \times\left[\frac{(G \times G) \times G}{\{((g, g), g)\}}\right]\right)\left(\epsilon^{A} \times b\right) \\
=\left[\frac{(I \times G) \times((I \times G) \times(G \times G))}{\left\{\left(\left(i^{\prime}, g_{1}\right),\left(\left(i^{\prime}, g_{2}\right),\left(g_{2}, g_{1}\right)\right)\right)\right\}}\right]\left[\frac{((I \times G) \times(G \times G)) \times G}{\{((i, \theta(i)),(g, g)), g)\}}\right] b \\
=\left[\frac{(I \times G) \times G}{\{((i, \theta(i)), \theta(i))\}}\right] b
\end{aligned}
$$

running over $i, i^{\prime} \in I$ and $g, g_{1}, g_{2} \in G$. A similar manipulation of the right-hand side of the asserted equality yields the same expression. So the asserted equality holds. Similar calculations show that

$$
\hat{v}(a) \circ \hat{\sigma}\left({ }_{F} \operatorname{ind}_{I}^{\alpha}\right)=\left[\frac{(F \times I) \times F}{\{((\alpha(i), i), \alpha(i))\}}\right] a=\hat{\sigma}\left({ }_{F} \operatorname{ind}_{I}^{\alpha}\right)_{\circ} \hat{v}\left({ }_{I} \operatorname{res}_{F}^{\alpha}(a)\right) .
$$

Using the same techniques, we obtain $\hat{v}(d) \circ \hat{\sigma}\left({ }_{I} \operatorname{res}_{F}^{\alpha}\right)=\left[\frac{(I \times F) \times I}{\{((i, \alpha(i)), i)\}}\right] d$ and then

$$
\hat{v}\left({ }_{F} \operatorname{ind}_{I}^{\alpha}(d)\right)=\left[\frac{(F \times F) \times I}{\{((\alpha(i), \alpha(i)), i)\}}\right] d=\hat{\sigma}\left({ }_{F} \operatorname{ind}_{I}^{\alpha}\right)_{\circ} \hat{v}(d) 。 \hat{\sigma}\left({ }_{I} \operatorname{res}_{F}^{\alpha}\right) .
$$

By Lemma 5.4, any element of ${ }^{\oplus} A$ can be expressed as a sum of elements having the form $\hat{\sigma}\left({ }_{F} \operatorname{ind}_{I}^{\alpha}\right)_{\circ} \hat{v}(d)_{\circ} \hat{\sigma}\left({ }_{I} \operatorname{res}_{G}^{\theta}\right)$. When two elements $u, v \in{ }^{\oplus} A$ are expressed as such a sum, the relations in Lemma 5.5 enable us to express the product $u_{\circ} v$ as such a sum.

We now turn to a study of ${ }^{\oplus} A$-modules. Any ${ }^{\oplus} A$-module becomes an ${ }^{\oplus} R \mathcal{H}$ functor via $\hat{\sigma}$. That is to say, for an ${ }^{\oplus} A$-module $M$, we let ${ }^{\oplus} R \mathcal{H}$ act on $M$ such that $x m=\hat{\sigma}(x) m$ for $x \in{ }^{\oplus} R \mathcal{H}$ and $m \in M$. We write $M(G)=\mathrm{id}_{G}^{A} M$.

Proposition 5.6. Let $M$ be an ${ }^{\oplus} A$-module.

(1) (Restriction property.) Given $I, G \in \mathfrak{L}$, a homomorphism $\theta: I \rightarrow G$ and $b \in A(G)$, $m \in M(G)$, then

$$
\hat{v}\left({ }_{I} \operatorname{res}_{G}^{\theta}(b)\right) \cdot{ }_{I} \operatorname{res}_{G}^{\theta}(m)={ }_{I} \operatorname{res}_{G}^{\theta}(\hat{v}(b) m) .
$$


(2) (Frobenius relations.) Given $F, I \in \mathfrak{L}$, a homomorphism $\alpha: F \leftarrow I$ and $a \in A(F)$, $m \in M(I), b \in A(I), n \in M(F)$, then

$$
{ }_{F} \operatorname{ind}_{I}^{\alpha}\left(\hat{v}\left({ }_{I} \operatorname{res}_{F}^{\alpha}(a)\right) m\right)=\hat{v}(a) \cdot{ }_{F} \operatorname{ind}_{I}^{\alpha}(m), \quad{ }_{F} \operatorname{ind}_{I}^{\alpha}\left(\hat{v}(b) \cdot{ }_{I} \operatorname{res}_{F}^{\alpha}(n)\right)=\hat{v}\left({ }_{F} \operatorname{ind}_{I}^{\alpha}(b)\right) n .
$$

Proof. This follows immediately from the latest lemma.

Proposition 5.7 (Mackey product formula). Let $M$ be an ${ }^{\oplus} A$-module and let $G, U, V \in \mathfrak{L}$. Let $\alpha: G \leftarrow U$ and $\beta: G \leftarrow V$ be homomorphisms. Let $u \in A(U)$ and $w \in$ $M(V)$. Then

$$
\hat{v}\left({ }_{G} \operatorname{ind}_{U}^{\alpha}(u)\right) \cdot{ }_{G} \operatorname{ind}_{V}^{\beta}(w)=\sum_{\alpha(U) g \beta(V) \subseteq G}{ }_{G} \operatorname{ind}_{L_{g}}^{\alpha \gamma_{g}}\left(\hat{v}\left({ }_{L_{g}} \operatorname{res}_{U}^{\gamma_{g}}(u)\right) \cdot{ }_{L_{g}} \operatorname{res}_{V}^{\eta_{g}}(w)\right)
$$

where $U \stackrel{\gamma_{g}}{\longleftarrow} L_{g} \stackrel{\eta_{g}}{\longrightarrow} V$ is any pullback of $U \stackrel{\alpha}{\longrightarrow} G \stackrel{\beta}{\longleftarrow} V$ such that $L_{g} \in \mathfrak{L}$.

Proof. Using part (2) of Proposition 5.6, the proof of Proposition 3.1 adapts easily.

Following Bouc $[1,8.5 .5]$, we define an $A$-module to be an $R \mathcal{H}$-functor $\mathcal{M}$ equipped with, for each $F, G \in \mathfrak{L}$, an $R$-bilinear map $A(F) \times \mathcal{M}(G) \ni(a, m) \mapsto a \times$ $m \in \mathcal{M}(F \times G)$ satisfying the following three conditions:

Associativity: Given $F, G, H \in \mathfrak{L}$ and $a \in A(F), b \in A(G), n \in \mathcal{M}(H)$ then, via the canonical isomorphism $(F \times G) \times H \leftarrow F \times(G \times H)$, we have $(a \times b) \times n=$ $(F \times G) \times H$ iso $_{F \times(G \times H)}(a \times(b \times n))$.

Identity: Given $G \in \mathfrak{L}$ and $m \in \mathcal{M}(G)$ then, via the canonical isomorphism $G \leftarrow$ $1 \times G$, we have ${ }_{G}$ iso $_{1 \times G}\left(\epsilon^{A} \times m\right)=m$.

Functorality: Given morphisms $x: F^{\prime} \leftarrow F$ and $y: G^{\prime} \leftarrow G$ in $R \mathcal{C}_{\mathfrak{L}}$ and $a \in A(F)$, $m \in \mathcal{M}(G)$, then $(x \times y)(a \times m)=x a \times y m$.

Given $A$-modules $\mathcal{M}$ and $\mathcal{M}^{\prime}$, we define a morphism $f: \mathcal{M}^{\prime} \leftarrow \mathcal{M}$ to be a morphism of $R \mathcal{H}$-functors such that $f_{F \times G}(a \times m)=a \times f_{G}(m)$ for $F, G, a, m$ as above.

A result stated in Bouc [1, 8.6.1.5], proved in Romero [4, 2.11], can be interpreted as saying that the category of ${ }^{\oplus} A$-modules is equivalent to the category of $A$-modules. We shall recover that result by adapting the proofs of Theorems 4.1 and 4.4. For $F, G \in \mathfrak{L}$, we define

$$
\begin{aligned}
& \operatorname{des}_{(F \times G) \times G}={ }_{F} \operatorname{def}_{F \times \Delta(G)} \operatorname{res}_{(F \times G) \times G}=\left[\frac{F \times((F \times G) \times G)}{\{(f,((f, g), g))\}}\right], \\
& { }_{(F \times G) \times G} \operatorname{tin}_{F}={ }_{(F \times G) \times G} \operatorname{ind}_{F \times \Delta(G)} \inf _{F}=\left[\frac{((F \times G) \times G) \times F}{\{(((f, g), g), f)\}}\right]
\end{aligned}
$$


running over $f \in F$ and $g \in G$. After [4, 2.11], given an $A$-module $\mathcal{M}$, we define an $R$-bilinear map $A(F, G) \times \mathcal{M}(G) \ni(u, m) \mapsto u m \in \mathcal{M}(F)$ where

$$
u m={ }_{F} \operatorname{des}_{(F \times G) \times G}(u \times m) .
$$

The next result is part of $[1,8.6 .1 .5],[4,2.11]$.

Theorem 5.8 (Bouc, Romero.). Let $\mathcal{M}$ be an A-module. Equipping $\mathcal{M}$ with the above map $(u, m) \mapsto$ um, then $\bigoplus_{G} \mathcal{M}(G)$ becomes an ${ }^{\oplus} A$-module.

Proof. The argument is similar to that in the first paragraph of the proof of Theorem 4.1.

Now let $M$ be a ${ }^{\oplus} A$-module. For each $F, G \in \mathfrak{L}$, we define an $R$-bilinear map $A(F) \times M(G) \ni(a, m) \mapsto a \times m \in M(F \times G)$ such that

$$
a \times m=\left(_{(F \times G) \times G} \operatorname{tin}_{F}(a)\right) m .
$$

Lemma 5.9. With the notation above, $a \times m=\hat{v}\left({ }_{F \times G} \inf _{F}^{p_{1}}(a)\right){ }_{F \times G} \inf _{G}^{p_{2}}(m)$.

Proof. We have

$$
\begin{aligned}
& \hat{v}\left(_{F \times G} \inf _{F}^{p_{1}}(a)\right)=\left[\frac{((F \times G) \times(F \times G)) \times F}{\{(((f, g),(f, g)), f)\}}\right] a, \\
& \hat{\sigma}\left({ }_{F \times G} \inf _{G}^{p_{2}}\right)=\left[\frac{((F \times G) \times G) \times 1}{\{((f, g), g), 1)\}}\right] \epsilon^{A}
\end{aligned}
$$

running over $f \in F$ and $g \in G$. A direct calculation gives

$$
(F \times G) \times G \operatorname{tin}_{F}(a)=\hat{v}\left(_{F \times G} \inf _{F}^{p_{1}}(a)\right)_{\circ} \hat{\sigma}\left({ }_{F \times G} \inf _{G}^{p_{2}}\right) .
$$

Lemma 5.10. With the notation above, the statements of Lemmas 4.2 and 4.3 still hold after replacing $b$ with $m$.

Proof. Using Lemma 5.9 and part (1) of Proposition 5.6,

$$
\begin{aligned}
F_{F^{\prime} \times G^{\prime}} \operatorname{res}_{F \times G}^{\theta \times \phi}(a \times m) & =\hat{v}\left({ }_{F^{\prime} \times G^{\prime}} \operatorname{res}_{F \times G}^{\theta \times \phi} \inf _{F}^{p_{1}}(a)\right) \cdot{ }_{F^{\prime} \times G^{\prime}} \operatorname{res}_{F \times G}^{\theta \times \phi} \inf _{G}^{p_{2}}(m) \\
& =\hat{v}\left(_{F^{\prime} \times G^{\prime}} \inf _{F^{\prime}}^{p_{1}} \operatorname{res}_{F}^{\theta}(a)\right) \cdot_{F^{\prime} \times G^{\prime}} \inf _{G^{\prime}}^{p_{2}} \operatorname{res}_{G}^{\phi}(m)={ }_{F^{\prime}} \operatorname{res}_{F}^{\theta}(a) \times{ }_{G^{\prime}} \operatorname{res}_{G}^{\phi}(b) .
\end{aligned}
$$

Let $u={ }_{F \times G^{\prime}} \inf _{F}^{p_{1}}(a)$ and $w={ }_{F^{\prime} \times G} \inf _{G}^{p_{2}}(m)$. Arguing as in the proof of Lemma 4.3 but using Proposition 5.7 instead of Proposition 3.1, we have

$$
\begin{aligned}
F_{F^{\prime} \times G^{\prime}} \operatorname{ind}_{F \times G}^{\alpha \times \beta}(a \times b) & ={ }_{F^{\prime} \times G^{\prime}} \operatorname{ind}_{F \times G}^{\alpha \times \beta}\left(\hat{v}\left(_{F \times G} \operatorname{res}_{F \times G^{\prime}}^{1 \times \beta}(u)\right) \cdot{ }_{F \times G} \operatorname{res}_{F^{\prime} \times G}^{\alpha \times 1}(w)\right) \\
& \left.=\hat{v}\left({ }_{F^{\prime} \times G^{\prime}} \operatorname{ind}_{F \times G^{\prime}}^{\alpha \times 1}(u)\right) \cdot{ }_{F^{\prime} \times G^{\prime}} \operatorname{ind}_{F^{\prime} \times G}^{1 \times \beta}(w)\right)={ }_{F^{\prime}} \operatorname{ind}_{F}^{\alpha}(a) \times{ }_{G^{\prime}} \operatorname{ind}_{G}^{\beta}(m) .
\end{aligned}
$$


The next result is another part of $[1,8.6 .1 .5],[4,2.11]$.

Theorem 5.11 (Bouc, Romero.). Let $M$ be an ${ }^{\oplus} A$-module. Equipping $M$ with the above operation $\times$, then $G \mapsto M(G)$ becomes an A-module.

Proof. The identity and associativity axioms are easy consequences of Lemma 5.3 and part (1) of Proposition 5.6. The functorality axiom holds by an argument similar to part of the proof of Theorem 4.4 but with Lemma 5.10 in place of Lemmas 4.2 and 4.3.

Let $M$ and $M^{\prime}$ be ${ }^{\oplus} A$-modules. Regard $M$ and $M^{\prime}$ as $A$-modules by equipping them with the operation $\times$. Any ${ }^{\oplus} A$-map $F: M^{\prime} \leftarrow M$ restricts to maps $f_{G}$ : $M^{\prime}(G) \leftarrow M(G)$ which evidently comprise a morphism of $A$-modules. Conversely, given maps $f_{G}: M^{\prime}(G) \leftarrow M(G)$ comprising a morphism of $A$-modules, then the sum $\bigoplus_{G} f_{G}: M^{\prime} \leftarrow M$ is an ${ }^{\oplus} A$-map. We have shown that the constructions in Theorems 5.8 and 5.11 give rise to mutually inverse equivalences between the category of ${ }^{\oplus} A$-modules and the category of $A$-modules.

\section{THE SYNTHETIC ALGEBRA AS A QUOTIENT ALGEBRA}

Continuing to work with $\mathfrak{L}$ and $\mathcal{H}$ as before and with a Green $R \mathcal{H}$-functor $A$, we shall realize the synthetic algebra $\Lambda_{A}$ as a quotient of ${ }^{\oplus} A$. Hence, by inflation, any $\Lambda_{A}$-module can be regarded as an ${ }^{\oplus} A$-module or, equivalently, as an $A$-module.

Proposition 6.1. There is a locally unital algebra map $\rho:{ }^{\oplus} A \rightarrow E_{A}$ determined by the condition that, given $F, G \in \mathfrak{L}$ and $u \in A(F, G), b \in A(G)$, then

$$
\rho(u) b={ }_{F} \operatorname{des}_{(F \times G) \times G}(u \times b) .
$$

Proof. Let $M$ be the ${ }^{\oplus} A$-submodule of ${ }^{\oplus} A$ such that $M(G)=A(G, 1)$. The action of ${ }^{\oplus} A$ on $M$ is such that an element $u \in A(F, G) \subseteq{ }^{\oplus} A$ sends an element $w \in M(G) \subseteq$ $M$ to the element $u_{\circ} w \in M(F) \subseteq M$. Let $\ell:{ }^{\Delta} A \rightarrow M$ be the $R$-isomorphism that restricts to maps $A(G) \rightarrow M(G)$ given by

$$
\ell(b)={ }_{G \times 1} \text { iso }_{G}(b)=b \times \epsilon^{A}
$$

for $b \in A(G)$. Let ${ }^{\Delta} A$ become a ${ }^{\oplus} A$-module by transport from $M$ via $\ell$ and let $\rho$ : ${ }^{\oplus} A \rightarrow E_{A}$ be the associated representation. Thus

$$
\ell(\rho(u) b)=u_{\circ} \ell(b)
$$

Since the representation of $M$ is locally unital, $\rho$ is locally unital. We have

$$
\rho(u) b={ }_{F} \operatorname{iso}_{F \times 1}\left(u_{\circ G \times 1} \operatorname{iso}_{G}(b)\right)={ }_{F} \operatorname{iso}_{F \times 1} \operatorname{des}_{(F \times G) \times(G \times 1)} \operatorname{iso}_{(F \times G) \times G}(u \times b) .
$$

A straightforward manipulation yields the asserted formula for $\rho(u) b$.

Lemma 6.2. With the notation above, $\sigma=\rho_{\circ} \hat{\sigma}$. 
Proof. We must show that $\rho(\hat{\sigma}(x)) b=\sigma(x) b$ for all $F, G \in \mathfrak{L}$ and $x \in R B(F, G)$, $b \in A(G)$. By the definitions of $\rho$ and $\hat{\sigma}$, we have

$$
\rho(\hat{\sigma}(x)) b={ }_{F} \operatorname{des}_{(F \times G) \times G}\left({ }_{(F \times G) \times 1} \text { iso }_{F \times G}(x) \times{ }_{G} \text { iso }_{G}\right)\left(\epsilon^{A} \times b\right) .
$$

We may assume that $x$ is transitive. Hence, by Remark 2.2, we can write $x=$ ${ }_{F} \operatorname{ind}_{I}^{\alpha} \operatorname{res}_{G}^{\theta}$ with $I \in \mathfrak{L}$. Then

$$
{ }_{(F \times G) \times 1} \operatorname{iso}_{F \times G}(x)=\left[\frac{(F \times G) \times 1}{\{((\alpha(i), \theta(i)), 1)\}}\right]
$$

running over $i \in I$. Therefore, running over $i, f, g, g^{\prime}$ in $I, F, G, G$, respectively,

$$
\begin{aligned}
\rho(\hat{\sigma}(x)) b & =\left[\frac{F \times((F \times G) \times G)}{\{(f,((f, g), g))\}}\right]\left[\frac{((F \times G) \times G) \times(1 \times G)}{\left\{\left(\left((\alpha(i), \theta(i)), g^{\prime}\right),\left(1, g^{\prime}\right)\right)\right\}}\right]\left(\epsilon^{A} \times b\right) \\
& =x b=\sigma(x) b .
\end{aligned}
$$

Lemma 6.3. With the notation above, $v=\rho_{\circ} \hat{v}$.

Proof. Given $b, b^{\prime} \in A(G)$, then $\rho(\hat{v}(b)) b^{\prime}={ }_{G} \operatorname{des}_{(G \times G) \times G} \operatorname{ind}_{G \times G}^{\delta_{G} \times 1}\left(b \times b^{\prime}\right)$. By direct calculation, $\rho(\hat{v}(b)) b^{\prime}=b b^{\prime}=v(b) b^{\prime}$.

Theorem 6.4. We have $\rho_{A}\left({ }^{\oplus} A\right)=\Lambda_{A}$.

Proof. This follows from Lemmas 5.4, 6.2, 6.3 and Proposition 3.3.

The latest theorem and the preceding two lemmas say that we have a commutative diagram of locally unital algebra maps as depicted, the vertical map being surjective.

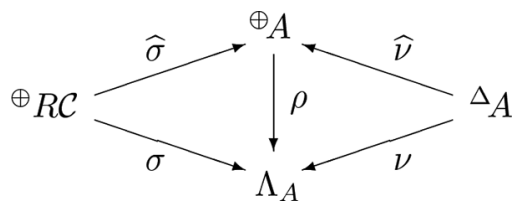

Regarding ${ }^{\Delta} A$ as a $\Lambda_{A}$-module via the inclusion $\Lambda_{A} \hookrightarrow E_{A}$ and regarding ${ }^{\Delta} A$ as an ${ }^{\oplus} A$-module via $\rho$, then the $\Lambda_{A}$-module structure inflates to the ${ }^{\oplus} A$-module structure. In the next section, we shall be examining module structure in two special cases, and we shall be making use of the next result.

Proposition 6.5. Suppose that $R$ is a field and that $A(G)$ is finite-dimensional for each $G \in \mathfrak{L}$. Then every simple $\Lambda_{A}$-module occurs in ${ }^{\Delta} A$.

Proof. A theorem of Green [3, Section 6.2] asserts that letting $\Gamma$ be a unital ring and letting $i$ be an idempotent of $\Gamma$, then the condition $T \cong i S$ characterizes a bijective correspondence between the isomorphism classes of simple $i \Gamma i$-modules $T$ 
and the isomorphism classes of simple $\Gamma$-modules $S$ satisfying $i S \neq 0$. To apply the theorem, we shall have to replace $\Lambda_{A}$ with a unital extension. Let $\Lambda=\Lambda_{A}$ and, for each $F, G \in \mathfrak{L}$, let $\Lambda(F, G)=\Lambda_{A}(F, G)$. As noted in Proposition 3.3, we have $\Lambda=\bigoplus_{F, G} \Lambda(F, G)$. Abusing notation, let us write elements of the direct product $\prod_{F, G} \Lambda(F, G)$ as infinite sums $\sum_{F, G} \lambda_{F, G}$ with $\lambda_{F, G} \in \Lambda(F, G)$. Let $\widetilde{\Lambda}$ be the $R$-submodule of $\prod_{F, G} \Lambda(F, G)$ consisting of those sums $\sum_{F, G} \lambda_{F, G}$ such that, for each $H \in \mathfrak{L}$, there are only finitely many $K \in \mathfrak{L}$ such that $\lambda_{H, K} \neq 0$ or $\lambda_{K, H} \neq 0$. Extending the multiplication operation on $\Lambda$ in the evident way, $\tilde{\Lambda}$ becomes a unital algebra over $R$ with unity element $\sum_{G} v\left(1_{A(G)}\right)$. Every $\Lambda$-module $M$ extends uniquely to a $\widetilde{\Lambda}$-module $\widetilde{M}$. Moreover, there is a bijective correspondence $S \leftrightarrow \widetilde{S}$ between the isomorphism classes of simple $\Lambda$-modules $S$ and the isomorphism classes of simple $\widetilde{\Lambda}$-modules $\widetilde{S}$. Given an idempotent $i \in \Lambda$, then $i \Lambda i=i \widetilde{\Lambda} i$. Therefore, by the above theorem of Green, the condition $T \cong i S$ characterizes a bijective correspondence between the isomorphism classes of simple $i \Lambda i$-modules $T$ and the isomorphism classes of simple $\Lambda$-modules $S$ satisfying $i S \leq 0$.

Let $S$ be a simple $\Lambda$-module. Choose an element $G \in \mathfrak{L}$ such that $S(G) \neq 0$. Choose an idempotent $i \in \Lambda(G, G)$ such that $i S(G) \neq 0$. We have $i \Lambda i=i \Lambda(G, G) i$ and $i S=i S(G)$. Also, $i^{\Delta} A=i A(G)$, which is finite-dimensional, say, $n=\operatorname{dim}_{R}\left(i^{\Delta} A\right)$. As $i \Lambda i$-modules by left multiplication, $i \Lambda i$ embeds in $i E_{A} i$. As an algebra, $i E_{A} i$ is isomorphic to the algebra of $n \times n$ matrices over $R$. As an $i \Lambda i$-module, $i E_{A} i$ is isomorphic to the direct sum of $n$ copies of $i^{\Delta} A$. But $i S$ occurs in $i \Lambda i$. Hence, iS occurs in $i E_{A} i$. By the Krull-Schmidt Theorem, $i S$ occurs in $i^{\Delta} A$. Therefore, thanks to the conclusion of the previous paragraph, $S$ occurs in ${ }^{\Delta} A$.

\section{THE MODULAR CHARACTER FUNCTOR}

For the two Green categories $\mathcal{G}_{\infty}$ and $\mathcal{G}_{G}$ discussed in Examples 2.6 and 2.7, we consider the Green functor $\mathbb{K} A_{\mathbb{F}}$. In both cases, we classify the simple modules of the synthetic algebra, and we show that they all have multiplicity 1 in ${ }^{\Delta} \mathbb{K} A_{\mathbb{F}}$.

Most of this section is concerned with the case of the Green category $\mathcal{G}_{\infty}$. As a Green $\mathbb{K} \mathcal{G}_{\infty}$-functor, let $A_{\infty}=\mathbb{K} A_{\mathbb{F}}$. We write the synthetic algebra as $\Lambda_{\infty}=\Lambda_{A_{\infty}}$. Given $G \in \mathfrak{K}_{\infty}$, we can understand the modular character of a finite-dimensional $\mathbb{F} G$-module to have values in $\mathbb{K}$. Thus, the elements of the algebra $\mathbb{K} A_{\mathbb{F}}(G)$ can be regarded as $G$-stable functions $G\left(p^{\prime}\right) \rightarrow \mathbb{K}$, where $G\left(p^{\prime}\right)$ denotes the $G$-set of $p^{\prime}$ elements of $G$. For $g \in G\left(p^{\prime}\right)$, let $e_{g}^{G}$ be the element of $\mathbb{K} A_{\mathbb{F}}(G)$ such that, given $g^{\prime} \in$ $G\left(p^{\prime}\right)$, then $e_{g}^{G}\left(g^{\prime}\right)=1$ if $g={ }_{G} g^{\prime}$ while $e_{g}^{G}\left(g^{\prime}\right)=0$ otherwise. We have direct sum decompositions

$$
{ }^{\Delta} A_{\infty}=\bigoplus_{G \in \mathfrak{K}_{\infty}} \mathbb{K} A_{\mathbb{F}}(G), \quad \mathbb{K} A_{\mathbb{F}}(G)=\bigoplus_{g \in G G\left(p^{\prime}\right)} \mathbb{K} e_{g}^{G}
$$

where the notation indicates that $g$ runs over representatives of the $p^{\prime}$-conjugacy classes. The next two results describe how induction and restriction act on the basis elements $e_{g}^{G}$. 
Lemma 7.1. Given $F, I \in \mathfrak{K}_{\infty}$, an injective homomorphism $\alpha: F \leftarrow I$ and $i \in I\left(p^{\prime}\right)$, then

$$
{ }_{F} \operatorname{ind}_{I}^{\alpha}\left(e_{i}^{I}\right)=\frac{\left|C_{F}(\alpha(i))\right|}{\left|C_{I}(i)\right|} e_{\alpha(i)}^{F}
$$

Proof. This follows from the formula

$$
{ }_{F} \operatorname{ind}_{I}^{\alpha}(\psi)(f)=\frac{1}{|I|} \sum_{s \in F: s_{f} \in \alpha(I)} \psi\left({ }^{s} f\right)
$$

where $\psi \in \mathbb{K} A_{\mathbb{F}}(I)$ and $f \in F\left(p^{\prime}\right)$.

Lemma 7.2. Given $I, G \in \mathfrak{K}$, a homomorphism $\theta: I \rightarrow G$ and $g \in G\left(p^{\prime}\right)$, then

$$
{ }_{I} \operatorname{res}_{G}^{\theta}\left(e_{g}^{G}\right)=\sum_{i \in_{I} I\left(p^{\prime}\right): \theta(i)={ }_{G} g} e_{i}^{I} .
$$

Proof. This follows from the fact that ${ }_{I} \operatorname{res}_{G}^{\theta}(\chi)(i)=\chi(\theta(i))$ for $\chi \in \mathbb{K} A_{\mathbb{F}}(G)$ and $i \in I\left(p^{\prime}\right)$.

Consider a set $\mathcal{N}$ of positive integers coprime to $p$ such that $\mathcal{N}$ is closed under taking multiples. Let ${ }^{\Delta} A_{\mathcal{N}}$ be the $\mathbb{K}$-submodule of ${ }^{\Delta} A_{\infty}$ spanned by those basis elements $e_{g}^{G}$ of ${ }^{\Delta} A_{\infty}$ such that $|\langle g\rangle| \in \mathcal{N}$. Given $I \in \mathfrak{K}_{\infty}$ and $i \in I\left(p^{\prime}\right)$ such that $e_{i}^{I} \in$ ${ }^{\Delta} A_{\mathcal{N}}$ then, in the notation of Lemma 7.1, ${ }_{F} \operatorname{ind}_{I}\left(e_{i}^{I}\right) \in{ }^{\Delta} A_{\mathcal{N}}$. Given $G \in \mathfrak{K}_{\infty}$ and $g \in$ $G\left(p^{\prime}\right)$ such that $e_{g}^{G} \in{ }^{\Delta} A_{\mathcal{N}}$ then, in the notation of Lemma 7.2, ${ }_{I} \operatorname{res}_{G}^{\theta}\left(e_{g}^{G}\right) \in{ }^{\Delta} A_{\mathcal{N}}$. We have shown that ${ }^{\Delta} A_{\mathcal{N}}$ is a $\sigma\left(\mathbb{K} \mathcal{G}_{\infty}\right)$-submodule of ${ }^{\Delta} A_{\infty}$. Plainly, ${ }^{\Delta} A_{\mathcal{N}}$ is a $v\left({ }^{\Delta} A_{\infty}\right)$ submodule of ${ }^{\Delta} A_{\infty}$. So ${ }^{\Delta} A_{\mathcal{N}}$ is a $\Lambda_{\infty}$-submodule of ${ }^{\Delta} A_{\infty}$.

Let $n$ be a positive integer coprime to $p$. We define a $\Lambda_{\infty}$-module $S_{n}$ as follows. As a $\mathbb{K}$-module, $S_{n}$ has a basis consisting of elements $s_{g}^{G}$ such that $G \in \mathfrak{K}_{\infty}$ and $g \in$ $G\left(p^{\prime}\right)$ with $|\langle g\rangle|=n$. Two basis elements $s_{g}^{G}$ and $s_{g^{\prime}}^{G^{\prime}}$ are understood to be equal if and only if $G=G^{\prime}$ and $g={ }_{G} g^{\prime}$. We allow $\Lambda_{\infty}$ to act on $S_{n}$ such that the following three conditions hold:

(1) Given $F \in \mathfrak{K}_{\infty} \ni I \ni i$ and injective $\alpha: F \leftarrow I$, then $\sigma\left({ }_{F} \operatorname{ind}_{I}^{\alpha}\right)\left(s_{i}^{I}\right)=\frac{\left|C_{F}(\alpha(i))\right|}{\left|C_{I}(i)\right|} s_{\alpha(i)}^{F}$.

(2) Given $I \in \mathfrak{K}_{\infty} \ni G \ni g$ and $\theta: I \rightarrow G$, then $\sigma\left({ }_{I} \operatorname{res}_{G}^{\theta}\right)\left(s_{g}^{G}\right)=\sum_{i \in I\left(p^{\prime}\right):|\langle i\rangle|=n, \theta(i)={ }_{G} g} s_{i}^{I}$.

(3) Given $i \in I \in \mathfrak{K}_{\infty} \ni I^{\prime} \ni i^{\prime}$ then $v\left(e_{i}^{I}\right)\left(s_{i^{\prime}}^{I^{\prime}}\right)= \begin{cases}s_{i}^{I} & \text { if } I=I^{\prime} \text { and } i={ }_{I} i^{\prime}, \\ 0 & \text { otherwise. }\end{cases}$

We must show that the three equations defining the action are consistent. Choose a set $\mathcal{N}$ as above such that $n$ is a minimal element of $\mathcal{N}$ with respect to division. We define a $\mathbb{K}$-map

$$
\pi_{\mathcal{N}}^{n}:{ }^{\Delta} A_{\mathcal{N}} \rightarrow S_{n}
$$


such that, given $G \in \mathfrak{K}_{\infty}$ and $g \in G\left(p^{\prime}\right)$ with $|\langle g\rangle| \in \mathcal{N}$, then

$$
\pi_{\mathcal{N}}^{n}\left(e_{g}^{G}\right)= \begin{cases}s_{g}^{G} & \text { if }|\langle g\rangle|=n, \\ 0 & \text { otherwise. }\end{cases}
$$

Using Lemmas 7.1 and 7.2, it is easy to check that $\pi_{\mathcal{N}}^{n}$ is surjective and commutes with the actions of $\sigma\left({ }_{I} \operatorname{res}_{G}^{\theta}\right)$ and $\sigma\left({ }_{F} \operatorname{ind}_{I}^{\alpha}\right)$ and $v\left(e_{i}^{I}\right)$. Therefore, the action of $\Lambda_{\infty}$ on $S_{n}$ is well defined and $\pi_{\mathcal{N}}^{n}$ is a $\Lambda_{\infty}$-epimorphism.

Lemma 7.3. For each positive integer $n$ coprime to $p$, the $\Lambda_{\infty}$-module $S_{n}$ is simple. Letting $n$ run over the positive integers coprime to $p$, the $S_{n}$ are mutually nonisomorphic.

Proof. Let $s \in S_{n}-\{0\}$. We are to show that $\Lambda_{\infty} s=S_{n}$. Let $G \in \mathfrak{K}_{\infty}$ and $g \in G\left(p^{\prime}\right)$ such that $|\langle g\rangle|=n$ and the coefficient $\lambda$ of $s_{g}^{G}$ in $s$ is nonzero. Let $I=\langle i\rangle$ be a cyclic group in $\mathfrak{K}$ with order $|I|=n$. Let $\theta: I \rightarrow G$ be the homomorphism such that $\theta(i)=$ $g$. We have

$$
s_{i}^{I}=v\left(e_{i}^{I}\right) \sigma\left({ }_{I} \operatorname{res}_{G}^{\theta}\right) s_{g}^{G}=\lambda^{-1} v\left(e_{i}^{I}\right) \sigma\left({ }_{I} \operatorname{res}_{G}^{\theta}\right) v\left(e_{g}^{G}\right) s \in \Lambda_{\infty} s .
$$

Let $F \in \mathfrak{K}_{\infty}$ and $f \in F\left(p^{\prime}\right)$ such that $|\langle f\rangle|=n$. Let $\alpha: F \leftarrow I$ be the homomorphism such that $\alpha(i)=f$. Then

$$
s_{f}^{F}=\frac{\left|C_{I}(i)\right|}{\left|C_{F}(f)\right|} \sigma\left({ }_{F} \operatorname{ind}_{I}^{\alpha}\right)\left(s_{i}^{I}\right) \in \Lambda_{\infty} s .
$$

But $s_{f}^{F}$ is an arbitrary basis element of $S_{n}$. So $\Lambda_{\infty} s=S_{n}$. The rider on mutual nonisomorphism is obvious. of ${ }^{\Delta} A_{\infty}$.

The next result gives a complete description of the composition structure

Theorem 7.4. We have ${ }^{\Delta} A_{\emptyset}=\{0\}$. Letting $\bar{\emptyset}$ denote the set of positive integers coprime to $p$, then ${ }^{\Delta} A_{\bar{\emptyset}}={ }^{\Delta} A_{\infty}$. Given a set $\mathcal{N}$ of positive integers coprime to $p$ such that $\mathcal{N}$ is closed under taking multiples then, for any minimal element $n \in \mathcal{N}$ with respect to division, we have an exact sequence of $\Lambda_{\infty}$-modules

$$
0 \rightarrow{ }^{\Delta} A_{\mathcal{N}-\{n\}} \rightarrow{ }^{\Delta} A_{\mathcal{N}} \rightarrow S_{n} \rightarrow 0
$$

In particular, the simple factors of the $\Lambda_{\infty}$-module ${ }^{\Delta} A_{\infty}$ are the $\Lambda_{\infty}$-modules $S_{n}$, where $n$ runs over the positive integers coprime to $p$. Each $S_{n}$ occurs with multiplicity 1 in ${ }^{\Delta} A_{\infty}$.

Proof. In our above discussion of the well defined $S_{n}$, the choice of $\mathcal{N}$ was subject only to the condition that $n$ is a minimal element of $\mathcal{N}$. We have $\operatorname{ker}\left(\pi_{\mathcal{N}}^{n}\right)={ }^{\Delta} A_{\mathcal{N}-\{n\}}$.

Finally, we can state and prove a classification theorem for the simple $\Lambda$-modules. 
Theorem 7.5. Letting $n$ run over the positive integers coprime to $p$, then $S_{n}$ runs, without repetitions, over the isomorphism classes of simple $\Lambda_{\infty}$-modules.

Proof. This follows from Proposition 6.5 and Theorem 7.4.

We now turn to the Green category $\mathcal{G}_{G}$ introduced in Example 2.7. As a Green $\mathbb{K} \mathcal{G}_{G}$-functor, let $A_{G}=\mathbb{K} A_{\mathbb{F}}$. We write $\Lambda_{G}=\Lambda_{A_{G}}$. We have

$$
{ }^{\Delta} A_{G}=\bigoplus_{I / \underline{I} \in \mathfrak{K}_{G}} A(I / \underline{I}), \quad A(I / \underline{I})=\bigoplus_{i I \in_{I / I} I / \underline{I}\left(p^{\prime}\right)} \mathbb{K} e_{i \underline{I}}^{I / \underline{I}}
$$

Lemma 7.1 implies that, given $a \in G$ and $H / \underline{H}, I / \widehat{I} \in \mathfrak{K}_{G}$ such that $H \geq{ }^{a} I$ and $\underline{H} \geq{ }^{a} \underline{I}$, then

$$
{ }_{H / \underline{H}} \operatorname{ind}_{I / \underline{I}}^{c(a)}\left(e_{i \underline{I}}^{I / I}\right)=\frac{\left|C_{H / \underline{H}}\left({ }^{a} i \cdot \underline{H}\right)\right|}{\left|C_{I / \underline{I}}(i \underline{I})\right|} e_{a_{i} \cdot \underline{H}}^{H / \underline{H}} .
$$

Lemma 7.2 implies that, given $u \in G$ and $I / \underline{I}, K / \underline{K} \in \mathfrak{K}_{G}$ such that ${ }^{u} I \leq K$ and ${ }^{u} \underline{I} \leq \underline{K}$, then

$$
{ }_{I / \underline{I}} \operatorname{res}_{K / \underline{K}}^{c(u)}\left(e_{k \underline{K}}^{K / \underline{K}}\right)=\sum_{i \underline{I} \epsilon_{I / \underline{I}} I / \underline{I}\left(p^{\prime}\right): u_{i} \cdot \underline{K}={ }_{K / \underline{K}} k \underline{K}} e_{i \underline{I}}^{I / \underline{I}} .
$$

We define a $p^{\prime}$-pair for $G$ to be a pair $(a A, A)$ where $A \leq G$ and $a A$ is a $p^{\prime}$ element of $N_{G}(A) / A$. We allow $G$ to act componentwise on the set of $p^{\prime}$-pairs for $G$. Given a $p^{\prime}$-pair $(a A, A)$ for $G$, we define a $\mathbb{K}$-module $S_{a A, A}$ with a basis consisting of elements $s_{i I}^{I / I}$ where $i \underline{I} \in I / \underline{I} \in \mathfrak{K}_{G}$ and $\underline{I}=A$ and $i \underline{I}=a A$. Two basis elements $s_{i I}^{I / I}$ and $s_{i^{\prime} I^{\prime}}^{I^{\prime}}$ are understood to be equal if and only if $I / \underline{I}=I^{\prime} / \underline{I}^{\prime}$ and $i I={ }_{I / \underline{I}} i^{\prime} I^{\prime}$. Note that $S_{a A, A}^{-}=S_{a^{\prime} A^{\prime}, A^{\prime}}$ if and only if $(a A, A)=_{G}\left(a^{\prime} A^{\prime}, A^{\prime}\right)$. We let $\Lambda_{G}$ act on $S_{a A, A}$ such that the following four conditions hold:

(1) Given $a \in G$ and $H / \underline{H} \in \mathfrak{K}_{G} \ni I / \underline{I} \in i \underline{I}$ such that $H \geq{ }^{a} I$ and $\underline{H}={ }^{a} \underline{I}$, then

$$
\sigma\left({ }_{H / \underline{H}} \operatorname{ind}_{I / \underline{I}}^{c(a)}\right)\left(s_{i \underline{I}}^{I / \underline{I}}\right)=\frac{\left|C_{H / \underline{H}}\left({ }^{a} i \cdot \underline{H}\right)\right|}{\left|C_{I / \underline{I}}(i \underline{I})\right|} s_{a_{i} \cdot \underline{H} \cdot \underline{H}}^{H / .}
$$

(2) Given $u \in G$ and $I \leq G$ and $k \underline{K} \in K / \underline{K} \in \mathfrak{K}_{G}$ such that $\underline{K} \unlhd^{u} I \leq K$, then

$$
\sigma\left({ }_{I / \underline{I}} \operatorname{res}_{K / \underline{K}}^{c(u)}\right)\left(s_{k \underline{K}}^{K / \underline{K}}\right)=s_{k^{u} . K^{u}}^{I / K^{u}} .
$$

(3) Given $u \in G$ and $I / \underline{I} \in \mathfrak{K}_{G} \ni K / \underline{K} \ni k \underline{K}$ such that ${ }^{u} I \leq K$ and ${ }^{u} \underline{I}<\underline{K}$ then

$$
\sigma\left({ }_{I / \underline{I}} \operatorname{res}_{K / \underline{K}}^{c(u)}\right)\left(s_{k \underline{K}}^{K / K}\right)=0 .
$$

(4) Given $i \underline{I} \in I / \underline{I} \in \mathfrak{K}_{G} \neq I^{\prime} / \underline{I}^{\prime} \ni i^{\prime} \underline{I}^{\prime}$, then

$$
v\left(e_{i \underline{I}}^{I / \underline{I}}\right)\left(s_{i^{\prime} \underline{I}^{\prime}}^{I^{\prime} / \underline{I}^{\prime}}\right)= \begin{cases}s_{i \underline{I}}^{I / \underline{I}} & \text { if } I / \underline{I}=I^{\prime} / \underline{I}^{\prime} \text { and } i \underline{I}={ }_{I / \underline{I}} i^{\prime} \underline{I^{\prime}}, \\ 0 & \text { otherwise. }\end{cases}
$$


Straightforward adaptations of arguments above show that $S_{a A, A}$ is a well defined simple $\Lambda_{G}$-module and that the following result holds.

Theorem 7.6. Letting $(a A, A)$ run over representatives of the $G$-orbits of $p^{\prime}$-pairs for $G$, then $S_{a A, A}$ runs, without repetitions, over the isomorphism classes of simple $\Lambda_{G^{-}}$ modules. Furthermore, each $S_{a A, A}$ occurs with multiplicity 1 in ${ }^{\Delta} A_{G}$.

\section{ACKNOWLEDGMENT}

This work was supported by Tübitak Scientific and Technological Research Funding Program 1001 under grant number 114F078.

\section{REFERENCES}

[1] Bouc, S. (2010). Biset Functors for Finite Groups. Lecture Notes in Math., Vol. 1990. Berlin: Springer-Verlag.

[2] Green, J. A. (1971). Axiomatic representation theory for finite groups. J. Pure Appl. Algebra 1:41-77.

[3] Green, J. A. (2007). Polynomial Representations of $G L_{n}$. 2nd ed. Lecture Notes in Math., Vol. 830. Berlin: Springer-Verlag.

[4] Romero, N. (2012). Simple modules over Green biset functors. J. Algebra 367:203-221.

[5] Thévenaz, J. (1988). Some remarks on $G$-functors and the Brauer morphism. J. Reine Angew. Math. 384:24-56.

[6] Webb, P. J. (2010). Stratifications of Mackey functors, II. J. K-Theory 6:99-170. 